\title{
Highlights of Transient Plume Impingement Model Validation and Applications
}

\author{
Michael S. Woronowicz ${ }^{1}$ \\ Stinger Ghaffarian Technologies, Inc., Greenbelt, Maryland 20770
}

This paper describes highlights of an ongoing validation effort conducted to assess the viability of applying a set of analytic point source transient free molecule equations to model behavior ranging from molecular effusion to rocket plumes. The validation effort includes encouraging comparisons to both steady and transient studies involving experimental data and direct simulation Monte Carlo results. Finally, this model is applied to describe features of two exotic transient scenarios involving NASA Goddard Space Flight Center satellite programs.

\section{Nomenclature}

\begin{tabular}{|c|c|c|}
\hline \multicolumn{3}{|c|}{ Variables } \\
\hline$A_{1}$ & $=$ & normalization factor \\
\hline$D$ & $=$ & generic exponent \\
\hline$d$ & $=$ & diameter \\
\hline$f$ & $=$ & $\begin{array}{l}\text { molecular distribution function, } \\
\text { also a correlation parameter }\end{array}$ \\
\hline $\boldsymbol{g}$ & $=$ & gravitational acceleration vector \\
\hline$M$ & $=$ & Mach number, also a generic flux \\
\hline$m$ & $=$ & mass \\
\hline$\dot{m}$ & $=$ & mass rate \\
\hline$n$ & $=$ & number density \\
\hline$\hat{\boldsymbol{n}}$ & $=$ & vector surface normal \\
\hline$p$ & $=$ & pressure, momentum flux \\
\hline$Q_{1}$ & $=$ & point source description \\
\hline$\dot{q}$ & $=$ & energy flux \\
\hline$R$ & $=$ & gas constant \\
\hline$r$ & $=$ & distance magnitude \\
\hline$S$ & $=$ & speed ratio $\beta u_{e}$ \\
\hline$T$ & $=$ & temperature \\
\hline$t$ & $=$ & time variable \\
\hline$u_{e}$ & $=$ & vector bulk exit velocity \\
\hline$V, v$ & $=$ & vector velocity variables \\
\hline$v$ & $=$ & velocity magnitude \\
\hline$w$ & $=$ & $s \cos \phi$ \\
\hline$x$ & $=$ & vector position variable \\
\hline$y$ & $=$ & $s \cos \phi_{\mathrm{e}}$ \\
\hline$z$ & $=$ & $\alpha-w$ \\
\hline$\alpha$ & - & $\beta r / t$ \\
\hline$\beta$ & $=$ & $1 / \sqrt{2 R T}$ \\
\hline
\end{tabular}

${ }^{1}$ Chief Engineer, Modeling and Contamination Analysis Section, 7701 Greenbelt Rd., Greenbelt, Maryland 20770. Senior Member, AIAA. 

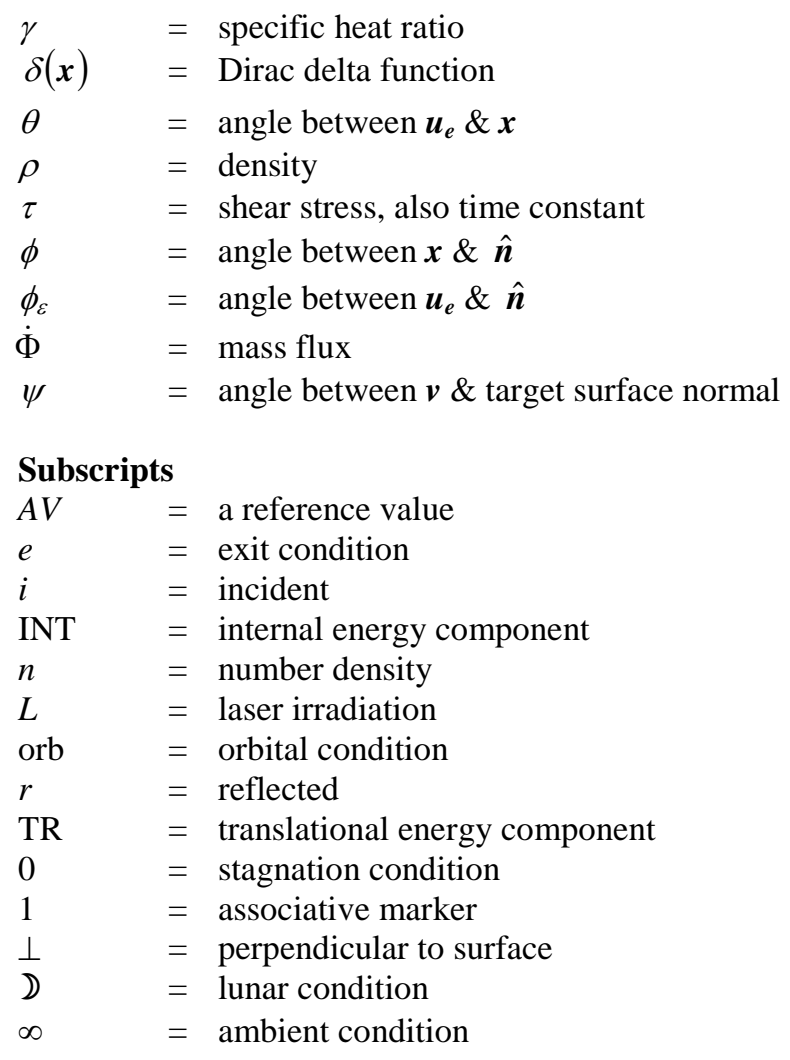

\section{Introduction}

$\mathrm{T}$

The analysis and simulation of gases expanding from sources such as rocket nozzles into vacuum, or the effects plumes from these sources create when they interact with solid surfaces, present a considerable challenge to the scientific and engineering communities. As a plume expands into vacuum, density levels, and hence collision rates, decrease rapidly by many orders of magnitude. The main difficulty lies in accurately describing a flowfield extending from continuum flow at the nozzle exit, through the transition regime, and reaching free molecule behavior within a relatively short distance downstream.

For thrusters, flow at the nozzle exit is usually characterized by high exit velocities and relatively high Mach numbers. Even in regions where significant intermolecular collision rates occur, relative velocity levels are low and little thermal scattering occurs normal to the mainly radial streamlines. Such observations lead one to consider describing the expansion under certain circumstances using free molecule (FM) theory.

Under such assumptions, a model was created by solving the Boltzmann equation for a point source with a Lambertian thermal velocity distribution superimposed on a bulk, convective exit velocity. This paper describes a number of comparisons of model results with experiment and direct simulation Monte Carlo applications, both steady and transient. Example cases involving thrusters, molecular beams, and pulsed laser ablation will be presented. Along with ongoing validation efforts, novel applications involving satellite programs at NASA Goddard Space Flight Center will be discussed.

\section{Model Development}

To develop this model, the collisionless Boltzmann equation had been solved for distribution function $f$ due to a directionally-constrained point source $Q_{1}$ meant to describe directed flow from a nozzle exit over $2 \pi$ steradians centered on the source normal. ${ }^{1}$ 


$$
\begin{aligned}
& \frac{\partial f}{\partial t}+\boldsymbol{v} \cdot \frac{\partial f}{\partial \boldsymbol{x}}+\boldsymbol{g} \cdot \frac{\partial f}{\partial \boldsymbol{v}} \approx \frac{\partial f}{\partial t}+\boldsymbol{v} \cdot \frac{\partial f}{\partial \boldsymbol{x}}=Q_{1} ; \quad Q_{1}=\frac{2 \beta^{4}}{A_{1} \pi} \delta(\boldsymbol{x}) \dot{m}(t)(\boldsymbol{v} \cdot \hat{\boldsymbol{n}}) \exp \left(-\beta^{2}\left(\boldsymbol{v}-\boldsymbol{u}_{\mathrm{e}}\right)^{2}\right) \\
& A_{1} \equiv e^{-s^{2} \cos ^{2} \phi_{\mathrm{e}}}+\sqrt{\pi} s \cos \phi_{\mathrm{e}}\left(1+\operatorname{erf}\left(s \cos \phi_{\mathrm{e}}\right)\right) .
\end{aligned}
$$

$Q_{1}$ represents directed flow from a Lambertian, thermal velocity distribution superimposed on convective exit velocity $\boldsymbol{u}_{\mathrm{e}}$ for mass flow rate $\dot{m}, \beta \equiv 1 / \sqrt{2 R T}$, and speed ratio $s \equiv \beta u_{\mathrm{e}}$. The influence of gravitational acceleration $\boldsymbol{g}$ is typically neglected for the applications of interest based on characteristic length scales and time-offlight estimates. Normal $\hat{\boldsymbol{n}}$ represents the orientation of a local starting surface element, and $\boldsymbol{v} \cdot \hat{\boldsymbol{n}}$ emphasizes the imposed directional constraint. Generally $\boldsymbol{u}_{\mathrm{e}}$ is not aligned with $\hat{\boldsymbol{n}}$, the angle between the two being defined by $\phi_{\mathrm{e}}$ (Fig. 1). Local angle $\phi$ is measured between variable position $\boldsymbol{x}$ (distance $r$, experiencing local velocity $\boldsymbol{v}$ ) and $\hat{\boldsymbol{n}}$, and angle $\theta$ is measured between $\boldsymbol{u}_{\mathrm{e}}$ and $\boldsymbol{x}$.
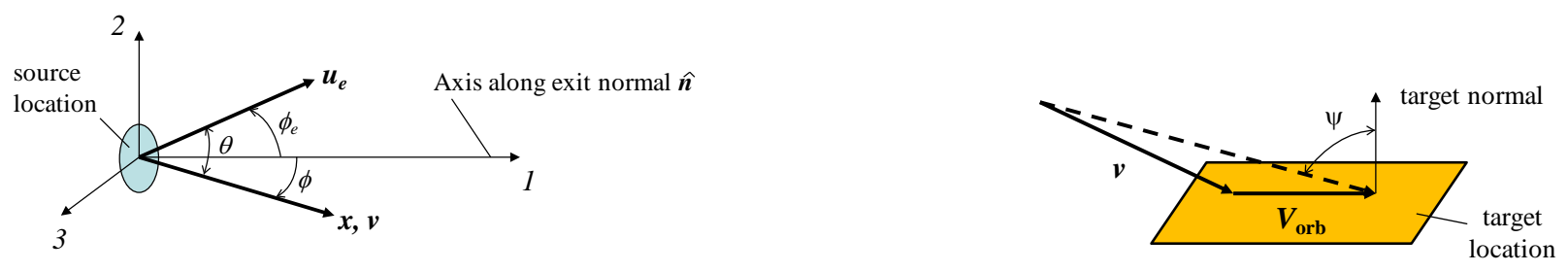

FIGURE 1. Schematic representation of various quantities and angles used in analytical model.

\section{A. Steady Source}

The distribution of various flowfield quantities is found by integrating successive velocity moments of the resulting distribution function. In particular, the steady-state density $\rho$, mass flux $\dot{\Phi}$, incident normal momentum flux $\tilde{p}_{\perp}$, and translational energy flux $\dot{q}_{\mathrm{TR}}$ are given by:

$$
\begin{gathered}
\rho(\boldsymbol{x}, t)=\frac{\beta \dot{m} \cos \phi}{A_{1} \pi r^{2}} e^{w^{2}-s^{2}}\left\{(\alpha+w) e^{-z^{2}}+\left(\frac{1}{2}+w^{2}\right) \sqrt{\pi} \operatorname{erfc} z\right\}, \\
\dot{\Phi}(\boldsymbol{x}, t)=\frac{\dot{m} \cos \phi \cos \psi}{A_{1} \pi r^{2}} e^{w^{2}-s^{2}}\left\{\left(\alpha^{2}+\alpha w+w^{2}+1\right) e^{-z^{2}}+\left(\frac{3}{2}+w^{2}\right) \sqrt{\pi} w \operatorname{erfc} z\right\}, \\
\tilde{p}_{\perp}(\boldsymbol{x}, t)=\frac{\dot{m} \cos \phi \cos \psi}{\beta A_{1} \pi r^{2}} e^{w^{2}-s^{2}}\left\{\left(\alpha^{3}+\alpha^{2} w+\alpha w^{2}+w^{3}+\frac{5}{2} w+\frac{3}{2} \alpha\right) e^{-z^{2}}+\left(\frac{3}{4}+3 w^{2}+w^{4}\right) \sqrt{\pi} \operatorname{erfc} z\right\},
\end{gathered}
$$

and

$$
\begin{aligned}
& \dot{q}_{\mathrm{TR}}(x, t)=\frac{\dot{m} \cos \phi \cos \psi}{2 \beta^{2} A_{1} \pi r^{2}} e^{w^{2}-s^{2}} \\
& \quad \times\left\{\left(\alpha^{4}+\alpha^{3} w+\alpha^{2} w^{2}+\alpha w^{3}+w^{4}+2 \alpha^{2}+\frac{7}{2} \alpha w+\frac{9}{2} w^{2}+2\right) e^{-z^{2}}+\left(\frac{15}{4}+5 w^{2}+w^{4}\right) \sqrt{\pi} w \operatorname{erfc} z\right\} .
\end{aligned}
$$


The normalization factor may be written as

$$
A_{1} \equiv e^{-y^{2}}+\sqrt{\pi} y(1+\operatorname{erf} y)
$$

Eq. (4) becomes incident normal momentum flux $p_{\perp}$ or shear stress $\tau$ when multiplied by $\cos \psi$ or $\sin \psi$, respectively. When the source-sink geometry is fixed, $\boldsymbol{V}_{\text {orb }}=0$. In these equations, $r$ and $\phi$ are components of position $\boldsymbol{x}$, measured from the centerline at the nozzle exit (Fig. 1), $z \equiv \alpha-w, \alpha \equiv \beta r / t, w \equiv s \cos \phi$, and $y \equiv s \cos \phi_{\mathrm{e}}$. Gas properties $\boldsymbol{u}_{\mathrm{e}}$, exit temperature $T$, and mass flow rate $\dot{m}$ are defined locally on the starting surface. For diatomic or polyatomic molecules with specific heat ratio $\gamma$, the internal energy flux $\dot{q}_{\text {INT }}$ is given by:

$$
\dot{q}_{\mathrm{INT}}(x)=\left(\frac{5-3 \gamma}{\gamma-1}\right) \frac{\dot{\Phi}(x)}{4 \beta^{2}} .
$$

In addition, Eqns. (2) - (4) may be combined to obtain local expressions for velocity $\boldsymbol{v}$ and translational temperature $T_{\mathrm{TR}}$ :

$$
\begin{gathered}
\boldsymbol{v}(\boldsymbol{x})=\frac{\dot{\Phi}(\boldsymbol{x})}{\rho(\boldsymbol{x})} ; \\
T_{\mathrm{TR}}(\boldsymbol{x})=\frac{1}{3 R}\left\{\frac{p_{\perp}(\boldsymbol{x})}{\rho(\boldsymbol{x})}-(\boldsymbol{v}(\boldsymbol{x}))^{2}\right\} .
\end{gathered}
$$

The total plume gas heat flux incident on a surface is given by $\dot{q}_{i}=\dot{q}_{\mathrm{TR}}+\dot{q}_{\mathrm{INT}}$. Reflected heat flux $\dot{q}_{r}$ is assumed to be carried away by diffusely reflected or desorbed plume molecules. The density of material is found by assuming the steady flux of molecules leaving a surface is equal to their rate of arrival, ${ }^{2}$ given by Eq. (3). One will recognize the resulting density distribution is consistent with Lambertian outgassing. After some algebra, one finds:

$$
\dot{q}_{r}(x)=\left(\frac{\gamma+1}{\gamma-1}\right) \frac{\dot{\Phi}(x)}{4 \beta_{r}^{2}}=\dot{q}_{\mathrm{INT}}(x)\left(\frac{\gamma+1}{5-3 \gamma}\right)\left(\frac{T_{r}}{T_{\mathrm{e}}}\right) .
$$

For reflected quantities, surface temperature $T_{r}$ is a transient quantity. Over time, the net heat flux should decrease as $T_{r}$ increases based on conduction and radiation heat transfer mechanisms. For operations having short duration, it may be reasonable to hold $T_{r}$ constant in order to compute pressure $p$ using Eq. (4), noting $p=p_{\perp}+p_{r}$.

Because FM models ignore intermolecular collisions, collisional effects will be absent from their solutions. These effects include ambient and self-scattered return flux, evolution of chemistry, and externally-developed thruster interaction shock waves. The flowfield emanating from this point source is only valid where $\boldsymbol{x} \cdot \hat{\boldsymbol{n}}>0$ due to the velocity constraint in Eq. (1). This last limitation is overcome by using local conditions from a starting surface that captures the expansion of gases expanding around the nozzle lip.

\section{B. Pulsed Source}

When $\dot{m}$ in Eq. (1) is described by a Dirac Delta function $\Delta m \delta(t)$, one may solve for successive moments of $f$ to find pulse-mode response expressions for various fluxes of interest.

$$
\rho(x, t)=\frac{2 \Delta m \alpha^{4} \cos \phi}{A_{1} \pi r^{3}} e^{w^{2}-s^{2}} e^{-z^{2}}
$$




$$
\dot{\Phi}(x, t)=\frac{\rho r}{t} \cos \psi ; \quad p_{\perp}(x, t)=\frac{\rho r^{2}}{t^{2}} \cos ^{2} \psi ; \quad \tau(x, t)=\frac{\rho r^{2}}{t^{2}} \sin \psi \cos \psi ; \quad \dot{q}_{\mathrm{TR}}(x, t)=\frac{\rho r^{3}}{2 t^{3}} \cos \psi
$$

\section{Model Development}

This model was initially used to help study Space Shuttle/Mir thruster interaction problems during docking maneuvers, where it provided checks on direct simulation Monte Carlo (DSMC) calculations. ${ }^{3}$ More recently, it has been used in support of a number of NASA-GSFC and -LaRC projects, with many successful comparisons to DSMC and experimental data. ${ }^{4-6}$ Applications include effusion (Mach number $M=0$ ), sonic orifices $(M=1$ ), and thruster plumes $(M>4)$.

\section{A. Sonic Orifice}

An experimental investigation had been conducted at NASA Goddard Space Flight Center (GSFC) to observe the angular distribution of molecular nitrogen venting through circular tubes of different diameters and mass flow rates. $^{4,7}$ The test was conducted at $T_{0}=290 \mathrm{~K}$ using GSFC's Thermal Vacuum Chamber 238 (C238), with background pressure levels $p_{\mathrm{C} 238}$ in the $10^{-4}-10^{-3}$ torr range during operations and driving pressures varying from 2-50 torr. These conditions ensured that the $\mathrm{N}_{2}$ issuing from the end of the tube would achieve sonic conditions, and for the lower driving pressure cases, $p_{\mathrm{C} 238}$ should not have interfered with the sonic expansion.

Measurements were made using a Baratron capacitance manometer fitted to flexible tubing, the open end of which was attached to a goniometer/crankwheel mechanism. It appeared that for cases having the lowest driving pressures ( $\dot{m}_{\mathrm{N}_{2}}$ below $0.02 \mathrm{~g} / \mathrm{s}, p_{\mathrm{C} 238}<4 \times 10^{-4}$ torr), data collected from the smallest cross-section duct ( $d=0.48$ $\mathrm{cm}$ ) might provide a suitable set of conditions for making comparisons with the free molecule plume model. Data for these cases meeting these criteria were normalized by their centerline values and used to develop a best fit angular distribution curve by the study's author. ${ }^{7}$ This fit had the following form:

$$
p \propto \cos ^{3} \theta
$$

A comparison of this angular distribution with plume model results for a single point source of molecular nitrogen at $M=1$ is presented in Fig. 2.

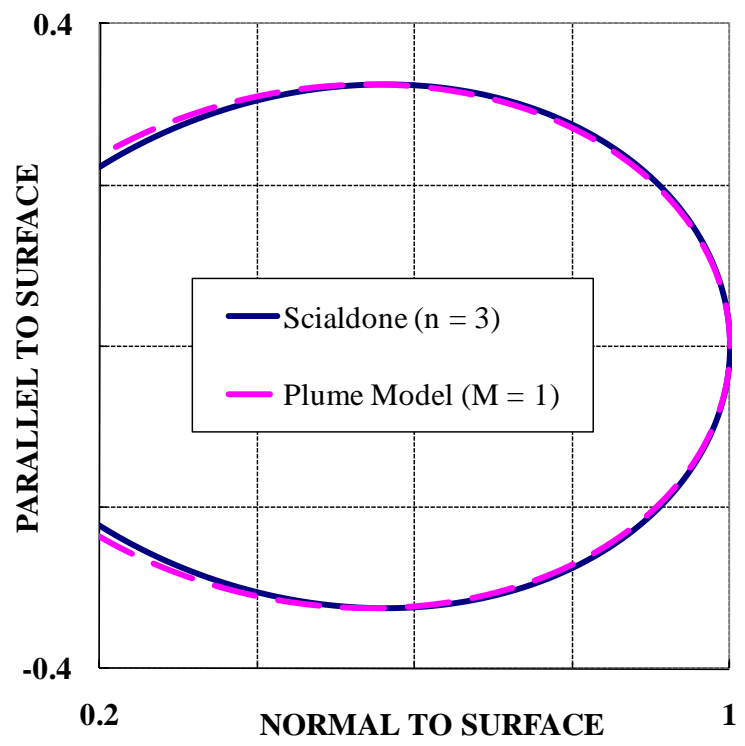

FIGURE 2. Comparison of Ref. 7 thruster model to FM sonic orifice flow for molecular nitrogen, Eq. (4). 


\section{B. CONTOUR Backflow}

On 15 August 2002, the NASA/Johns Hopkins University Applied Physics Laboratory (APL) Comet Nucleus Tour (CONTOUR) spacecraft was destroyed during conclusion of a solid rocket motor orbit transfer burn. ${ }^{8}$ A NASA Mishap Investigation Board (MIB) was established one week after loss of contact, and under its direction, a number of failure modes were investigated and revisited in greater detail. ${ }^{8}$ Ultimately, heating of the spacecraft due to plume radiation and convective impingement in the backflow regime was reasoned to be the first element of the proximate cause for its demise. ${ }^{8}$ In the course of the investigation, a computational fluid dynamics (CFD) solution for the near-field gaseous expansion produced by NASA-Marshall Space Flight Center (MSFC) was used to provide a starting surface for DSMC and free molecule plume modeling efforts to determine fluxes in the backflow regime. ${ }^{9}$

A comparison of model flowfield results is presented in Figs. 3a \& 3b. In the figures, the CONTOUR spacecraft (S/C) forebody is shown on the left, while the CFD starting surface is represented by the shadowed region to the right. In between are flowfield density contours for the plume impinging on the satellite from right to left. These exhibit a high level of agreement over three orders of magnitude. The free molecule contours consist not only of the direct contribution from the plume (at high speed ratios $s$ ), but surface reflected molecules as well $(s=0)$.
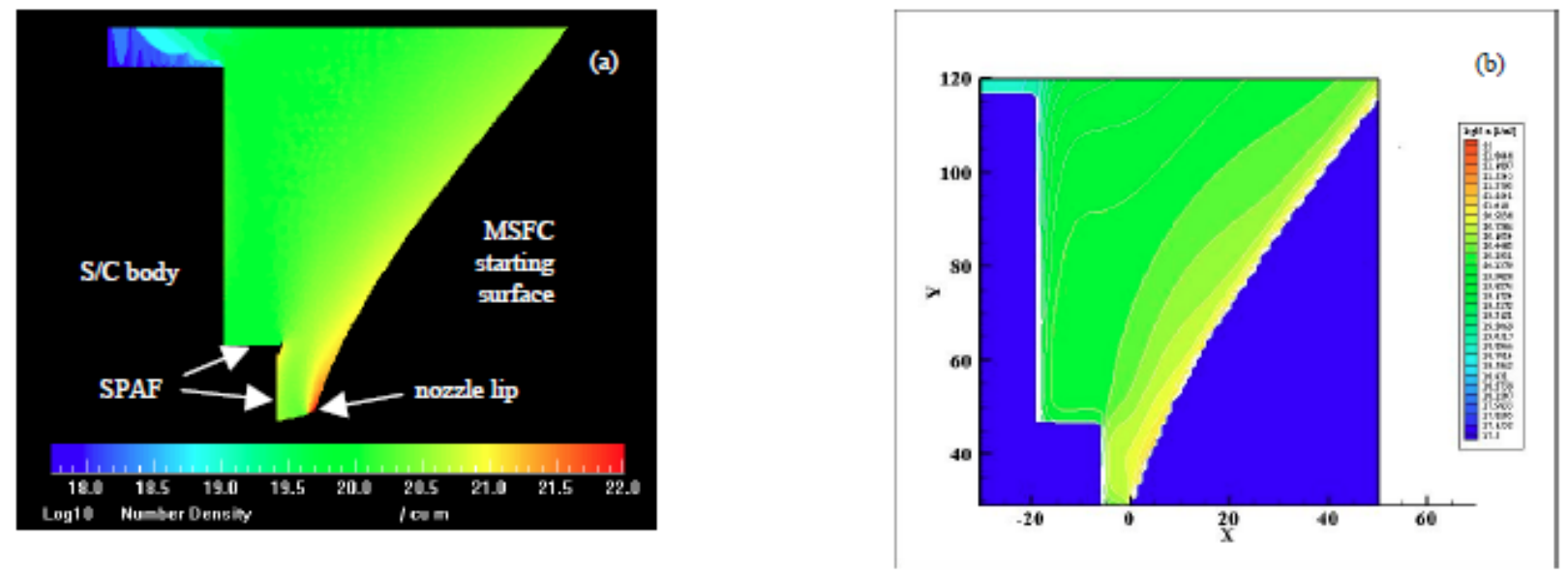

FIGURE 3. Comparison of steady DSMC/DS2V (a) and FM results (b),
logarithmic number density contour maps.

Sampling the flowfield axially at different radii revealed the high levels of agreement were maintained nearly everywhere, even by the S/C Payload Attachment Fitting (SPAF), except very close to the spacecraft forebody surface. In the DSMC solution, a radial Knudsen layer had developed where density increased as the surface was approached from a normal direction. In the free molecule model, mass conservation ensured that density levels would drop instead of increase there. Incident heat flux profiles across most of the forebody were similar, except the presence of a SPAF attachment ring in the DSMC geometry led to a localized intensity of heating that was absent from the free molecule calculation.

\section{Molecular Beam Time-of-Flight Data}

One novel feature of this plume model is its transient flow capability. When considering model validation, it is difficult to recreate highly-rarefied conditions using ground-based facilities due to demands for sufficient pumping capacity to eliminate the influence of vacuum chamber surface scattering on a thruster plume expansion. ${ }^{10,11}$ However, it is possible to compare limiting cases of such models to data collected during carefully executed experiments employing molecular beams (MB). In particular, a number of early experiments measured molecular time-of-flight (TOF) data to produce velocity distributions associated with low-density sources. ${ }^{12,13}$

In one such experiment, Miller \& Kusch measured TOF data for effusion $(M=0)$ of potassium and thallium at $900 \mathrm{~K}$ and confirmed the on-axis distribution of density was given by: 


$$
\rho \propto \alpha^{4} e^{-\alpha^{2}}
$$

Such a relationship is also given by Eq. (11) for pulse-mode flow for speed ratio $s=0$ and $\phi=0$ (Ref. 11 ).

In another molecular beam experiment incorporating a nozzle for their source, Anderson \& Fenn found for high Mach number TOF measurements along the nozzle centerline: ${ }^{13}$

$$
\rho \propto \alpha^{4} e^{-z^{2}}
$$

Again, this relationship is consistent with Eq. (11), but for $s>0$.

\section{Transient Pulsed Laser Ablation Simulation}

Bykov et al. modeled a transient pulsed laser ablation process for heating and vaporization of a niobium target with varying laser fluence levels $\left(4-6 \mathrm{~J} / \mathrm{cm}^{2}\right)$ of brief duration, using direct simulation Monte Carlo (DSMC) for the subsequent vapor release. ${ }^{11,14}$ It was noted that the response of vaporized effluent angular distributions to increasing heat inputs was to change from a spherical expansion to one more focused along the surface normal initially. Such distributions would relax to spherical expansions as time and rarefaction levels increased. ${ }^{14}$ A plot of centerline density function $f_{n}$ versus $\alpha$ was presented (Fig. 4), where

$$
f_{n} \equiv \frac{n t^{3}}{n_{A V} \tau_{L}}
$$

In Eq. (19) $n$ is the local number density, $n_{A V}$ is a reference value based on the total amount of removed mass (dependent upon laser fluence level), and $\tau_{L}$ is the 13 ns laser irradiation period. ${ }^{14}$ The $f_{n}$ curve for each laser fluence level features a maximum ( $\alpha \approx 0.8$ for $4 \mathrm{~J} / \mathrm{cm}^{2}, 1.15$ for $6 \mathrm{~J} / \mathrm{cm}^{2}$ ), and for large levels of $\alpha$ (short time periods) the functions decay rapidly.

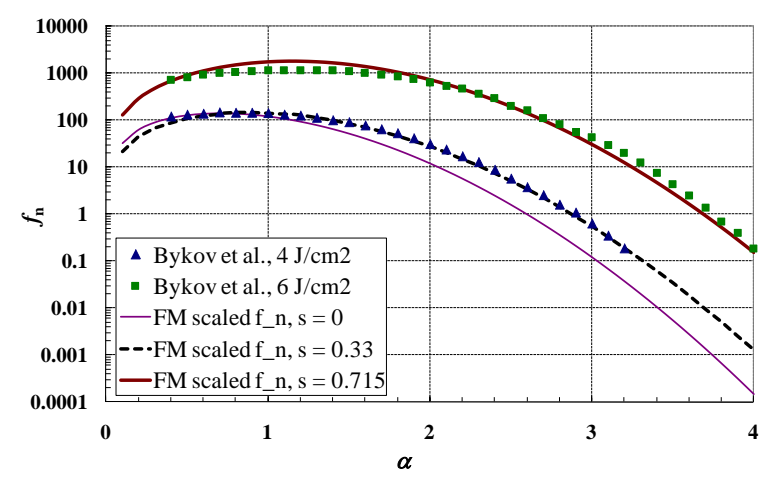

FIGURE 4. Comparison of Eq. (11) development with Ref. 14 DSMC simulations of transient PLA plume centerline responses for different heat flux inputs.

From Eq. (11), one can write an equivalent centerline variation:

$$
n t^{3} \propto f_{n} \propto \alpha e^{-z^{2}}
$$

which is maximized for 


$$
\alpha=\frac{s+\sqrt{s^{2}+2}}{2} .
$$

Not enough information is given in Ref. 14 to complete a direct comparison to their $f_{n}$ curves, but Eq. (21) indicates maxima shift from $\alpha=1 / \sqrt{2}$ for effusive flow to larger values for $s>0$, which would be consistent with the sort of energy transfer into more directed flows Ref. 14 authors observed for higher laser fluence levels. Substituting $s=0.33 \& 0.715$ in Eq. (20), corresponding to $\alpha$ values similar to those producing maximum $f_{n}$ values in the DSMC results listed above, model results provide fair matches for the $4 \& 6 \mathrm{~J} / \mathrm{cm}^{2}$ cases with arbitrary density scaling. This level of agreement extends over a wide range of time scales $(\propto 1 / \alpha)$ even though intermolecular collisions are responsible for deviations from effusive flow close to the model surface.

\section{Other Applications}

Over time, ongoing validation activities such as the examples included in the previous section have led to increasing confidence in using the molecular flow plume model as a tool to describe an unusual variety of plume interaction issues, particularly for NASA Goddard Space Flight Center spacecraft missions. Two applications featuring transient effects are briefly presented below.

\section{A. CloudSat-CALIPSO Interactions}

CloudSat and CALIPSO are the second and third elements of the Afternoon Constellation, or "A-Train", a joint effort between NASA and ESA to operate a heterogeneous group of scientific satellites in a coordinated effort to make a variety of near-simultaneous atmospheric measurements over an identical ground track. ${ }^{15,16}$ The A-Train operates in a sun-synchronous orbit at $705 \mathrm{~km}$ altitude. Each satellite's Attitude Control System (ACS) features either $1 \mathrm{lb}_{\mathrm{f}}$ or $1 \mathrm{~N}$ aft-facing monopropellant hydrazine thrusters. Thruster firing periods range from $7-100 \mathrm{~s}$. Plume species were based on 100 percent decomposition of high-purity grade monopropellant hydrazine with 65 percent ammonia decomposition, ${ }^{17}$ adding 0.5 percent mass fraction of water vapor as a system impurity. A nozzle exit velocity $V_{\mathrm{e}}=2.2 \mathrm{~km} / \mathrm{s}$ was assumed, with a mass flow rate $\dot{m}$ of $2.0 \mathrm{~g} / \mathrm{s}$ per $\mathrm{lb}_{\mathrm{f}}$ thrust.

TABLE 1. CloudSat Model ACS Plume Species Mass Fractions.

\begin{tabular}{|c|c|}
\hline Species & $\begin{array}{c}\text { Mass } \\
\text { Fraction }\end{array}$ \\
\hline $\mathrm{NH}_{3}$ & 0.247 \\
\hline $\mathrm{N}_{2}$ & 0.667 \\
\hline $\mathrm{H}_{2}$ & 0.081 \\
\hline $\mathrm{H}_{2} \mathrm{O}$ & 0.005 \\
\hline
\end{tabular}

The MSISE-90 atmospheric model ${ }^{18}$ generated estimates of atmospheric density for A-Train conditions of $n_{\infty} \approx$ $2.1 \times 10^{11}$ molecules $/ \mathrm{m}^{3}( \pm 5 \times)$, essentially made up of atomic oxygen. The calculated mean free path length associated with plume-ambient molecular scattering under such conditions is immense ( 7000 km). This estimate is only representative at an altitude of $705 \mathrm{~km}$; clearly there will be an overwhelming level of interaction in the lower reaches of the atmosphere, and the plume will not develop symmetrically with altitude.

Nevertheless, some program managers were concerned about the possibility that thruster operations by one satellite in this formation may create unwanted impacts on the function of others. CALIPSO trails CloudSat nominally by $15 \mathrm{~s}$, equating to a separation distance of roughly $112.5 \mathrm{~km}$. CloudSat employs a pair of $1 \mathrm{~N}$ thrusters, and ACS operations typically last $19 \mathrm{~s}$. Due to the separation distance, peak impingement fluxes on CALIPSO should be experienced roughly 50-70 s after a CloudSat ACS unit commences firing.

Due to the short ACS firing intervals relative to the time taken to travel distances separating A-Train spacecraft, it might not be adequate to assume steady conditions for determining surface flux couplings. It was decided to use Eqns. (2) - (10) in transient mode as a square wave to observe the development of CALIPSO impingement fluxes due to CloudSat ACS thruster operations. In addition to neglecting scattering occurring via ambient conditions at 
$705 \mathrm{~km}$ altitude, the effects of atmospheric density stratification with altitude and solar ionization have been neglected, making this study a first approximation to actual impingement behavior.

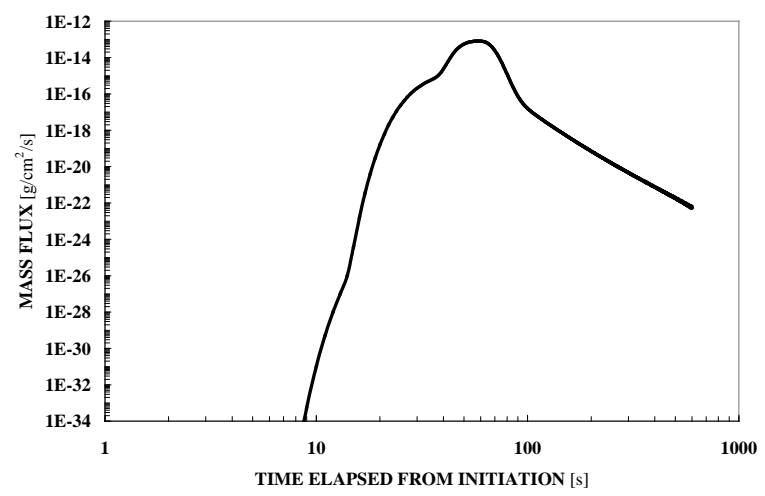

FIGURE 5. Transient mass flux experienced by CALIPSO due to single CloudSat ACS operation.

Results for total impingement mass flux $\dot{\Phi}$ (combination of $\mathrm{N}_{2}, \mathrm{H}_{2}, \mathrm{NH}_{3}$, and $\mathrm{H}_{2} \mathrm{O}$ as described in Ref. 17) experienced by CALIPSO due to CloudSat operations are presented in Fig. 5 above. The initial steep rise in $\dot{\Phi}$ is due to dominance of the directionally-independent wave-like term $\alpha \equiv \beta r / t$ in parameter $z$. However, as time progresses, the effect of bulk motion being directed downstream in the model causes a shift in this rise, manifested by the kink occurring at about 13 s elapsed time.

Another deviation in the transient mass flux impingement profile occurs around the period anticipated for peak fluxes to occur. This is due to a species separation effect. The transient effective molecular weight of the gases reaching CALIPSO's ram-facing surface is presented in Fig. 6 below.

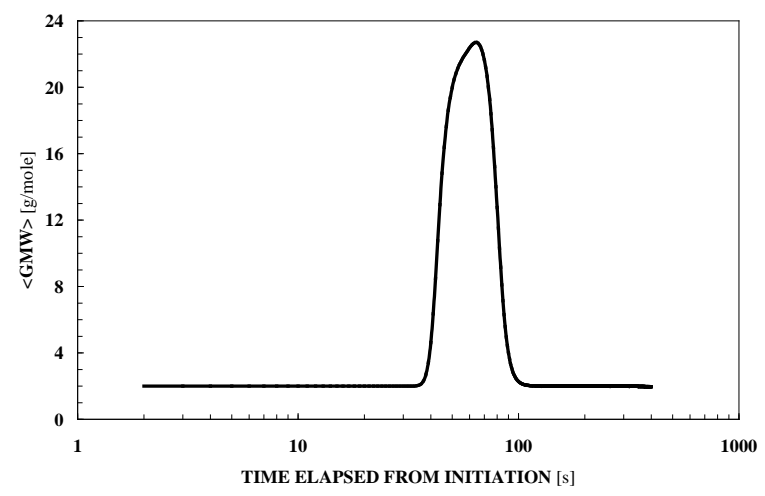

\section{FIGURE 6. Transient average molecular weight experienced by CALIPSO due to single CloudSat ACS operation.}

Before the pronounced bulge in Fig. 5 occurs, the gas consists almost entirely of molecular hydrogen. At high degrees of rarefaction, lightweight species will tend to spread fairly diffusely while heavier species remain more tightly focused around the plume axis, traveling more closely to the average bulk velocity. ${ }^{19}$ The peak effective molecular weight reaches $22.7 \mathrm{~g} /$ mole at $64 \mathrm{~s}$ elapsed time, versus an expected average molecular weight of 12.9 $\mathrm{g} / \mathrm{mole}$. At later times, heavier gases disappear and the residual ensemble consists of molecular hydrogen again. Notice how this gas appears to linger in Fig. 5 even though the thruster was only on for $19 \mathrm{~s}$. At its peak, the effect of firing two CloudSat thrusters over a $19 \mathrm{~s}$ interval produced a density of $7 \times 10^{-19} \mathrm{~g} / \mathrm{cm}^{3}$, an increase of roughly 12 percent over ambient levels.

Other observations for this application involved comparisons between mass flux and other fluxes. ${ }^{15}$ Among other things, higher velocity moment solutions featured steeper final decay rates as square wave solutions degenerated to those for a pulse source, Eqns. (11) - (15). In the pulse source solutions, every increase in velocity 
moment includes another power of $t^{-1}$. It was also noted that higher velocity moment peak fluxes occurred at slightly shorter elapsed intervals than lower ones. ${ }^{15}$

\section{B. LADEE - Surface-Scattered Flux Environment}

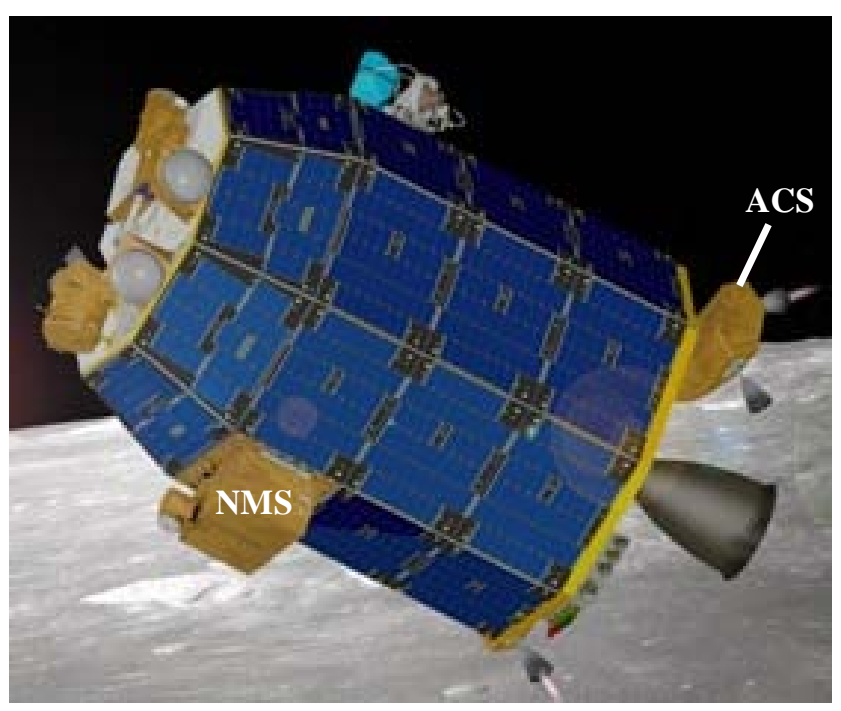

FIGURE 7. Artist's rendering of the Lunar Atmosphere Dust Environment Explorer.

The Lunar Atmosphere Dust Environment Explorer (LADEE) spacecraft is being designed for a mission featuring low altitude orbits of the Moon to take relevant ambient measurements before that environment becomes altered by future activities associated with human exploration (Fig. 7). Depending on orbital insertion timing, the observatory's mission could last over four months, beginning in $2013 .^{20}$ In some cases, the orbital altitude may dip as low as $20 \mathrm{~km}$ above the lunar surface. ${ }^{20}$ LADEE's instrument suite includes a sensitive Neutral Mass Spectrometer (NMS) which will be oriented in the spacecraft's ram direction to measure tenuous atmospheric constituents. Ambient density levels vary widely based on local solar illumination, but may exceed $10^{5}$ molecules $/ \mathrm{cm}^{3}$ during a lunar dawn. ${ }^{21}$ NMS is being designed to detect individual species concentrations at much lower levels, however.

LADEE's attitude control system features $5-\mathrm{lb}_{\mathrm{f}}$ bipropellant thrusters mounted in two canted pairs beneath the spacecraft's lower deck. ${ }^{20}$ If the ACS units are directed forward and toward the lunar surface, it is possible for individual species associated with their operations, reflected from the daytime lunar surface, to be quantitatively measured by the NMS as the observatory subsequently passes overhead. At lower altitudes, thruster plume fluxes to the lunar surface are maximized, as are reflected fluxes back to the spacecraft; also NMS may intercept peak species fluxes before lunar gravitational disturbances exert much influence. In this manner, NMS measurements could be used to test a variety of characteristics associated with this transient plume model, including evolution of species concentration (magnitudes, time-of-flight measurements, angular distribution, species separation effects), thruster exit conditions, or to infer local elements of lunar surface properties such as permeation or gas-surface interaction models.

LADEE will orbit the Moon in a variety of orientations and orbit altitudes. With surface values of $1737 \mathrm{~km}$ for lunar radius $R$, and a gravitational acceleration constant of $g_{\supset}=1.62 \mathrm{~m} / \mathrm{s}^{2}$, the orbital velocity at $20 \mathrm{~km}$ circular altitude should be $V_{\text {orb }}=1.67 \mathrm{~km} / \mathrm{s}^{21}$ A study was developed ${ }^{22}$ assuming a pair of these ACS thrusters facing forward and $20^{\circ}$ below the satellite velocity vector were operated for just one second using the pulsed source model. Under these conditions, it was considered that gravitational effects on the gas at this altitude and for an ACS bulk velocity of $V_{\mathrm{e}} \approx 3.0 \mathrm{~km} / \mathrm{s}$ could be neglected. ${ }^{22}$ Expected plume product nozzle exit temperature was $T_{\mathrm{e}} \approx 550 \mathrm{~K}$, and ACS plume product species are presented in Table 2 below. ${ }^{23}$ Interactions with a featureless lunar surface were modeled as impermeable, chemically-inert, and diffusely-reflecting. Such details allowed use of the plume model for $s=0$ conditions and a simple mass conservation statement for the surface-reflected elements. 
TABLE 2. Dominant expected LADEE ACS plume species mass fractions. ${ }^{23}$

\begin{tabular}{|c|c|}
\hline Species & $\begin{array}{c}\text { Mass } \\
\text { Fraction }\end{array}$ \\
\hline $\mathrm{N}_{2}$ & 0.426 \\
\hline $\mathrm{H}_{2} \mathrm{O}$ & 0.294 \\
\hline $\mathrm{CO}$ & 0.178 \\
\hline $\mathrm{CO}_{2}$ & 0.086 \\
\hline $\mathrm{H}_{2}$ & 0.016 \\
\hline
\end{tabular}

Under such conditions, results indicate LADEE's NMS could indeed detect surface-reflected ACS thruster plume gases (Fig. 8) with peak values occurring within a minute after that brief operation. Individual species responses are separated by molecular weight, with peak molecular hydrogen measurements in particular occurring appreciably more quickly than expected based on bulk velocity and geometric configuration (19-20 s). Reviewing the pulse source equations and noting that flux $\mathrm{M}$ varies as $t^{-\mathrm{D}}$, the time to reach a peak flux becomes

$$
t_{\max f l u x}=\frac{2 \beta r}{w\left(1+\sqrt{1+\frac{2 D}{w^{2}}}\right)} .
$$

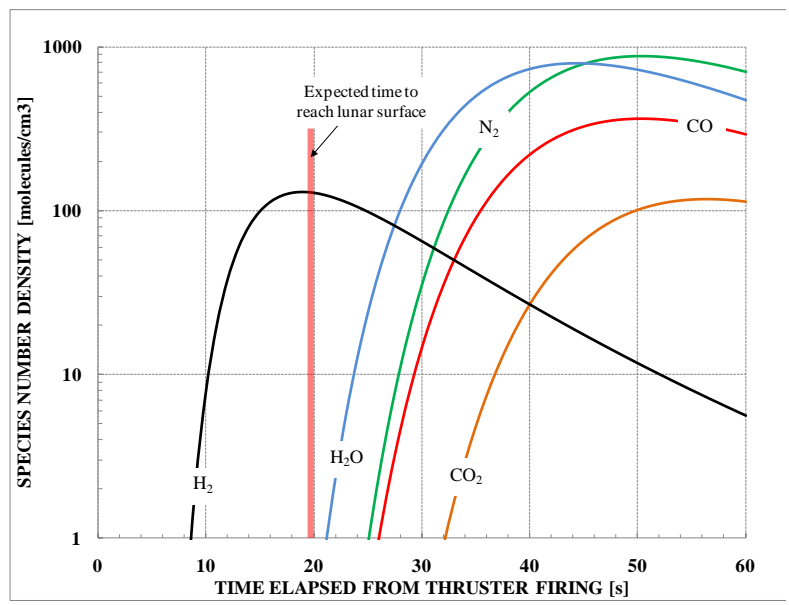

FIGURE 8. Predicted ACS species contributions measured by LADEE NMS, assumed conditions.

For $w=s$ on the plume centerline, $t_{\max \text { flux }} \rightarrow r / V_{\mathrm{e}}$ as $s \rightarrow \infty$. For any finite value of $s$ however, the time to reach maximum flux actually decreases due to the component of kinetic energy associated with the non-zero thermal distribution. In Fig. 8, the peak surface-reflected $\mathrm{H}_{2}$ concentration reaches NMS in less time than one would expect the average molecule to reach the surface! This effect was investigated further by artificially reducing $T_{\mathrm{e}}$ to $55 \mathrm{~K}$, which led to a tighter focus of gas tending to reach the lunar surface around $19 \mathrm{~s}$ and a delayed response reaching NMS compared to Fig. $8 .{ }^{22}$

\section{Concluding Remarks}

A number of results have been presented from an ongoing validation effort for a transient free molecule thruster plume model over a wide range of applications. Encouraging results have led the author to assist NASA Goddard Space Flight Center and other organizations assess increasingly complex scenarios associated with exotic scientific spacecraft missions. It is hoped that such efforts will allow these organizations to perform their activities with greater confidence and possibly enhance general understanding. 


\section{Acknowledgements}

The author gratefully acknowledges support from NASA Contract NNG07CA21C, especially Ms Sharon Straka, NASA-GSFC Code 546, and SGT, Inc.

\section{References}

${ }^{1}$ Woronowicz, M., "Development of a Novel Free Molecule Rocket Plume Model”, Proceedings of the $22^{\text {nd }}$ Intl. Symposium on Rarefied Gas Dynamics, Vol. No. 797, American Institute of Physics, Melville, New York, 2001, pp. 798-805.

${ }^{2}$ Bird, G. A., Molecular Gas Dynamics and the Direct Simulation of Gas Flows, Clarendon Press, Oxford, 1994, pp. 162-172.

${ }^{3}$ Woronowicz, Michael S., and Rault, Didier F.G., “On Plume Flowfield Analysis and Simulation Techniques”, AIAA Paper No. 94-2048, $6^{\text {th }}$ AIAA/ASME Joint Thermophysics and Heat Transfer Conference, Colorado Springs, CO, 20-23 June 1994.

${ }^{4}$ Woronowicz, M., "Further Studies Using A Novel Free Molecule Rocket Plume Model”, Proceedings of the $23^{\text {rd }}$ Intl. Symposium on Rarefied Gas Dynamics, Vol. 595, American Institute of Physics, Melville, New York, 2003, pp. 798-805.

${ }^{5}$ Chavis, Z., and Wilmoth, R., "Plume Modeling and Application to Mars 2001 Odyssey Aerobraking", Journal of Spacecraft and Rockets, Vol. 42, No. 3, 2005, pp. 450-6.

${ }^{6}$ Prince, H., et al., "Modeling Reaction-Control-System Effects on Mars Odyssey”, Journal of Spacecraft and Rockets, Vol. 42, No. 3, 2005, pp. 444-9.

${ }^{7}$ Scialdone, J. J., “Flow Fields of Low Pressure Vent Exhausts,” NASA TM 100738, July 1989.

${ }^{8}$ Bradley, T., et al., CONTOUR Mishap Investigation Board Report, National Aeronautics and Space Administration, Washington, DC, 31 May 2003, 64 pp.

${ }^{9}$ Woronowicz, M., "Solid Rocket Motor Backflow Analysis for CONTOUR Mishap Investigation”, Proceedings of the $24^{\text {th }}$ Intl. Symposium on Rarefied Gas Dynamics, Vol. 762, American Institute of Physics, Melville, New York, 2005, pp. 431-6.

${ }^{10}$ G. Dettleff, K. Plähn, “Experimental Investigation of Fully Expanding Free Jets and Plumes”, Proceedings of the $21^{\text {st }}$ International Symposium on Rarefied Gas Dynamics, Vol. 1, Cépaduès Éditions, Toulouse, 1999, pp. 60715.

${ }^{11}$ Woronowicz, M., "Simple Validation of Transient Plume Models Using Molecular Beam-Related Applications", Proceedings of the $26^{\text {th }}$ Intl. Symposium on Rarefied Gas Dynamics, Vol. 1084, American Institute of Physics, Melville, New York, 2009, pp. 565-70.

${ }^{12}$ R. C. Miller, P. Kusch, "Velocity Distribution in Potassium and Thallium Atomic Beams”, Physical Review, Vol. 99, No. 4, 1955, pp.1314-21.

${ }^{13}$ J. Anderson, J. Fenn, "Velocity Distributions in Molecular Beams from Nozzle Sources”, Physics of Fluids, Vol. 8, No. 5, 1965, pp. 780-7.

${ }^{14}$ N. Y. Bykov et al., "Modeling of Vapor Expansion under Pulsed Laser Ablation: Time-of-flight Data Analysis”, Proceedings of the $24^{\text {th }}$ Intl. Symposium on Rarefied Gas Dynamics, Vol. 762, American Institute of Physics, Melville, New York, 2005, pp. 373-378. 
${ }^{15}$ Woronowicz, M., “Selected Afternoon Constellation Transient Plume Impingement Model Results”, ESA $3^{\text {rd }}$ International Symposium on Formation Flying, Missions and Technologies, SP-654, European Space Agency, 2008.

${ }^{16}$ Woronowicz, M., “Transient Plume Impingement Analysis For Formation Flying Spacecraft”, Proceedings of the $25^{\text {th }}$ Intl. Symposium on Rarefied Gas Dynamics, Publishing House of the Siberian Branch of the Russian Academy of Sciences, 2007, pp. 853-8.

${ }^{17}$ Genovese, J., "Rapid Estimation of Hydrazine Exhaust Plume Interaction”, AIAA Paper No. 78-1091, AIAA/SAE 14 ${ }^{\text {th }}$ Joint Propulsion Conference, Las Vegas, Nevada, 25-27 July 1978.

${ }^{18}$ Hedin, A., "Extension of the MSIS Thermospheric Model into the Middle and Lower Atmosphere”, $J$. Geophys. Res., Vol. 96, 1991, pp. 1159-72.

${ }^{19}$ Koppenwallner, G., "Species Separation in Rocket Exhaust Plumes and Analytic Plume Flow Models", Rarefied Gas Dynamics $22^{\text {nd }}$ Intl. Symposium, Vol. No. 585, American Institute of Physics, Melville, New York, 2001, p. 797.

${ }^{20}$ Mecham, M., “Low Pass”, Aviation Week \& Space Technology, Vol. 172, No. 3, 18 January 2010, pp. 25-26.

${ }^{21}$ Heiken, G. et al., Lunar Sourcebook—A User's Guide to the Moon, Cambridge University Press, NY, 1991, pp. 28, 40-5.

${ }^{22}$ Woronowicz, M., “Transient Plume Model Testing Using LADEE Attitude Control System Operations”, $27^{\text {th }}$ Intl. Symposium on Rarefied Gas Dynamics, Vol. 1333, American Institute of Physics Conference Proceedings, Melville, New York, 2011, pp. 619-24.

${ }^{23}$ Trinks, H., Hoffman, R., "Experimental Investigation of Bipropellant Exhaust Plume Flowfield, Heating and Contamination, and Comparison with the CONTAM Computer Model Predictions”, AIAA Paper No. 83-1447, Montreal, QC, Jun. 1983. 


\section{Highlights of Transient Plume Impingement Model Validation and Applications}

Dr. Michael Woronowicz

Stinger Ghaffarian Technologies, Inc.

AIAA Thermophysics Conference

Sheraton Waikiki, Honolulu, HI

27-30 June 2011 


\section{Outline}

- Introduction

- Model Development

- Selected Model Comparisons

- Selected Unusual Satellite Applications

- Concluding Remarks 


\section{Introduction}

- Analysis and simulation of gases expanding into high vacuum present considerable challenges to engineering and scientific communities

- Examples for spacecraft environmental issues

- plume impingement (forces, heating)

- contamination of sensitive surfaces

- optical instrument apertures (mass flux, density)

- thermal control surfaces (mass flux)

- Flowfield passes from continuum to free-molecule regimes in relatively short distance 


\section{Introduction (2 of 2)}

- Existence of collisions may not substantially alter plume distribution from free-molecule description

- collision rates fall rapidly with distance from source

- majority of self-scattering collisions occur when faster molecules overtake slower ones on similar trajectories from source

- center-of-mass motion remains unchanged during collision

- Can consider modeling this regime using free molecule flow 


\section{Plume Model Formulation--Source}

- Find particular solution to collisionless Boltzmann equation for source $Q_{1}$ :

where

$$
\frac{\partial f}{\partial t}+\boldsymbol{v} \cdot \frac{\partial f}{\partial \boldsymbol{x}}+\boldsymbol{g} \cdot \frac{\partial f}{\partial \boldsymbol{v}}=Q_{1}
$$

and

$$
Q_{1} \equiv \frac{2 \beta^{4}}{A_{1} \pi} \delta(x) \dot{m}(t)|v \cdot \hat{\boldsymbol{n}}| \exp \left(-\beta^{2}\left(\boldsymbol{v}-\boldsymbol{u}_{\mathrm{e}}\right)^{2}\right)
$$

$$
A_{1} \equiv e^{-s^{2} \cos ^{2} \phi_{\mathrm{e}}}+\sqrt{\pi} s \cos \phi_{\mathrm{e}}\left(1+\operatorname{erf}\left(s \cos \phi_{\mathrm{e}}\right)\right)
$$




\section{Plume Model Development--Source}

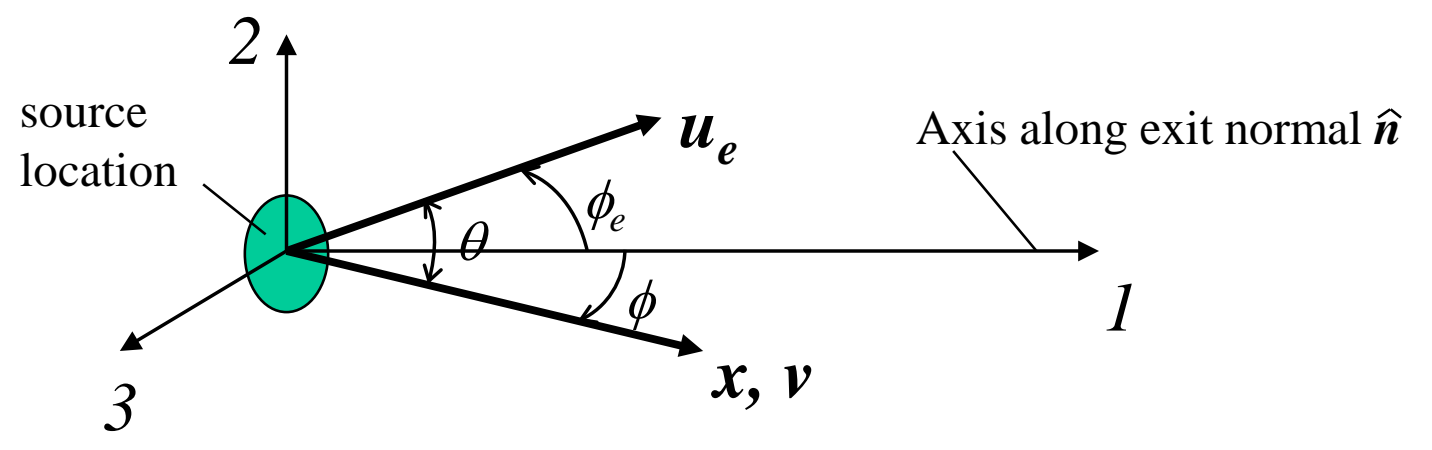

- Impingement angle with target surface given by $\psi$

- Simplifies for axisymmetric conditions

$$
\begin{aligned}
& -\phi_{\mathrm{e}}=0 \\
& -\phi=\theta
\end{aligned}
$$

- other definitions:

$$
s \equiv \beta u_{\mathrm{e}}=\frac{u_{\mathrm{e}}}{\sqrt{2 R T_{\mathrm{e}}}} ; \quad z \equiv \alpha-w ; \quad \alpha \equiv \beta r / t ; \quad w \equiv s \cos \theta
$$




\section{Model Development--Velocity Moments}

$$
\begin{array}{r}
\rho(x, t)=\frac{\beta \dot{m} \cos \phi}{A_{1} \pi r^{2}} e^{w^{2}-s^{2}}\left\{(\alpha+w) e^{-z^{2}}+\left(\frac{1}{2}+w^{2}\right) \sqrt{\pi} \operatorname{erfc} z\right\} \\
\dot{\Phi}(\boldsymbol{x}, t)=\frac{\dot{m} \cos \phi \cos \psi}{A_{1} \pi r^{2}} e^{w^{2}-s^{2}\left\{\left(\alpha^{2}+\alpha w+w^{2}+1\right) e^{-z^{2}}\right.} \\
\left.+\left(\frac{3}{2}+w^{2}\right) \sqrt{\pi} w \operatorname{erfc} z\right\}
\end{array}
$$

- Similar, but more complicated expressions for $p_{\perp}, \tau, \dot{q}_{\mathrm{TR}}, \dot{q}_{\mathrm{INT}}$

- Can also estimate

- diffuse, surface-reflected quantities $(s=0)$

- local average velocity, $<\mathrm{GMW}>$, temperature with suitable ratios 


\section{Model Development--Limitations}

- No inherent mechanism for gaseous collisional effects in free molecule flow

- Molecular scattering

- Chemical reactions

- In present application

- Neglect stratification of atmospheric density with altitude

- Possible influence of solar ionization

- Neglect physical presence of satellite bodies

- Consider this study as first approximation

- Could possibly be revisited if motivation existed 


\section{Model Development—Pulse}

- In plume model analytical development for this application, can also create velocity moments for case where mass flow rate is described by $\dot{m}=\Delta m \delta(t)$

$$
\begin{gathered}
\rho(x, t)=\frac{2 \Delta m \alpha^{4} \cos \phi}{A_{1} \pi r^{3}} e^{-(w-s)^{2}} e^{-z^{2}} ; \\
\dot{\Phi}(x, t)=\frac{\rho r}{t} \cos \psi ; \quad \dot{q}_{\mathrm{TR}}(x, t)=\frac{\rho r^{3}}{2 t^{3}} \cos \psi ; \\
p_{\perp}(x, t)=\frac{\rho r^{2}}{t^{2}} \cos ^{2} \psi ; \quad \tau(x, t)=\frac{\rho r^{2}}{t^{2}} \sin \psi \cos \psi
\end{gathered}
$$




\section{Outline}

- Introduction

- Model Development

- Selected Model Comparisons

- Steady

- Sonic Orifice

- CONTOUR SRM backflow

- Transient

- Molecular Beam Experiments

- Pulsed Laser Ablation

- Selected Satellite Applications

- Concluding Remarks 


\section{Sonic Orifice Comparison}

- Test performed at NASA-GSFC to measure density angular distribution

- $\mathrm{N}_{2}$ at various $p_{0}$, tube diameters

- Appears conditions closest to FM flow for lowest $p_{0}, d_{\mathrm{i}}$

- Results suggest

$$
\rho \propto \cos ^{3} \theta
$$

- Can compare to steady plume model result for $M=1$ 


\section{Sonic Orifice Comparison}

COMPARISON OF ANGULAR DISTRIBUTIONS FOR MOLECULAR FLOW EXPANSIONS

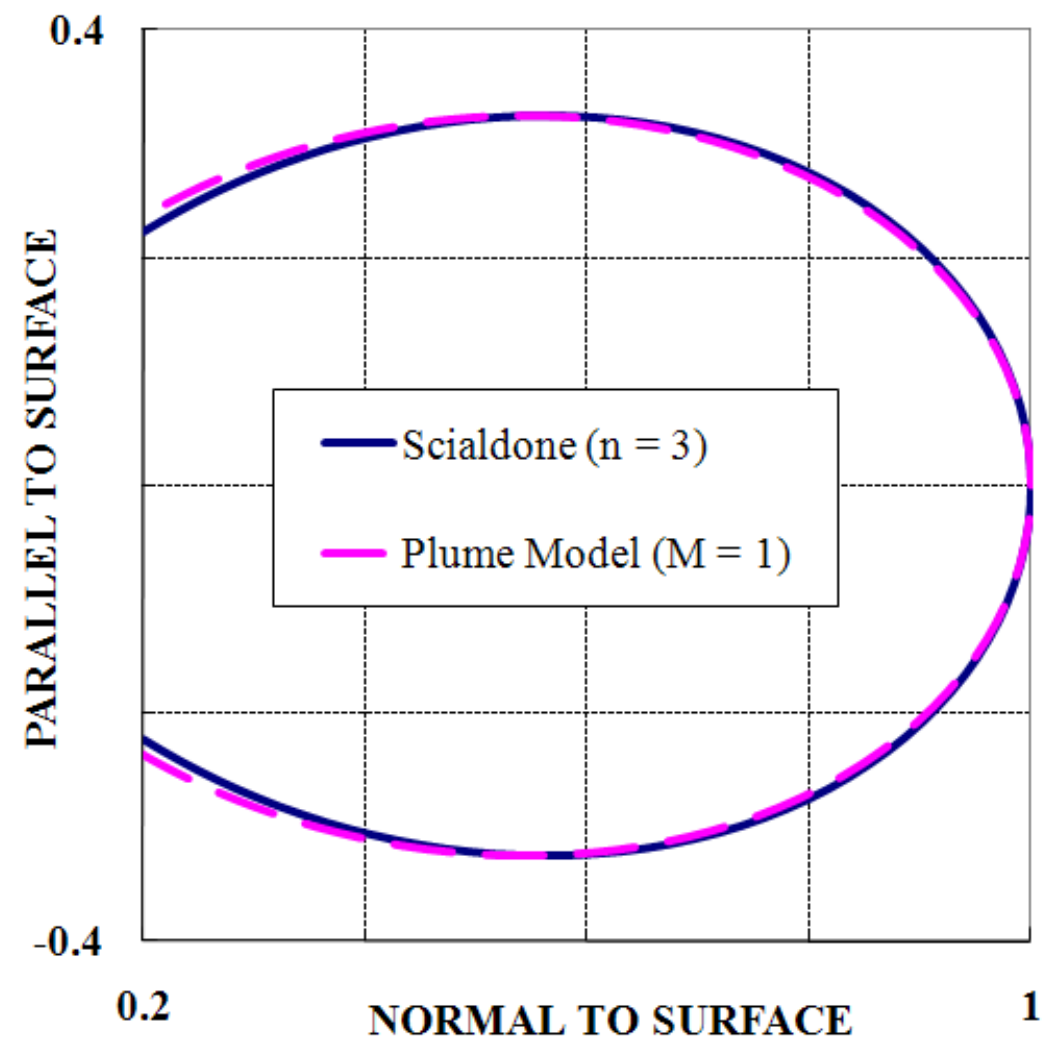




\section{CONTOUR SRM Backflow}

- CONTOUR spacecraft destroyed during conclusion of SRM orbit transfer burn; Mishap Investigation Board formed to determine proximate cause for this outcome

- NASA-MSFC supplied CFD starting surface for DSMC study of high-angle satellite surface convective heat input

- Simplified (gases only)

- Asked to compare using plume model

- FM flowfield result of superposition

- Free expansion

- Surface-reflected gases ( $s=0$, no deposition) 


\section{CONTOUR Model SRM Flowfields}
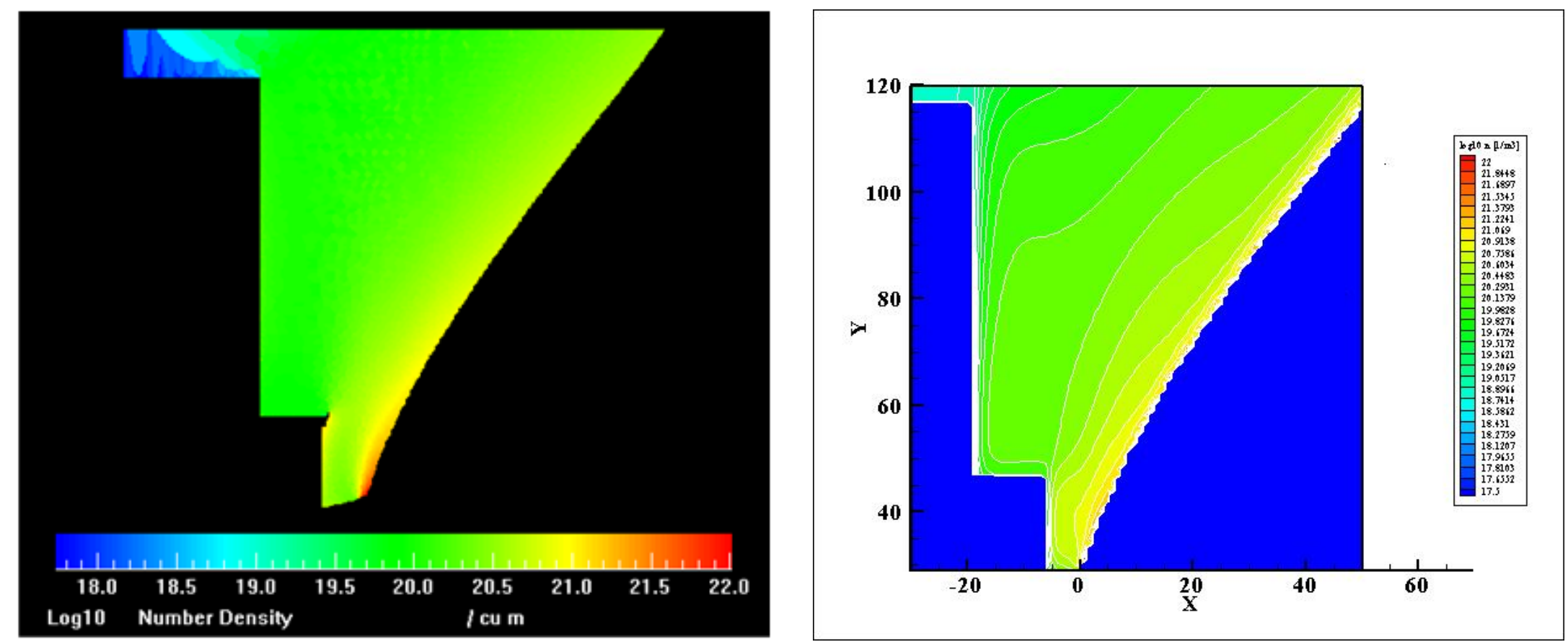

- Density contours, logarithmic scale 


\section{CONTOUR Model Comparison-Cmts.}

- Decent level of agreement over four orders of magnitude within flowfield

- Comparisons of surface fluxes also exhibit good agreement

- Pressure

- Convective heat flux levels

- Growth of Knudsen layer in DSMC model responsible for subtle differences

- DSMC contours increase as surface is approached closely; FM flowfield contours fall to free expansion values

- Buildups in surface fluxes displaced radially outward 


\section{Molecular Beam TOF}

- Transient model comparisons difficult to verify experimentally

- Difficult to eliminate effects due to vacuum chamber surface scattering for thruster plumes

- Exception: STG Facility, DLR-Göttingen

- Can compare limiting cases for plume model with carefully executed molecular beam (MB) experiments

- Time-of-flight (TOF) data used to produce velocity distributions associated with low-density sources

- Miller \& Kusch, effusion ( $s=0)$ of potassium and thallium @ $900 \mathrm{~K}$

- Anderson \& Fenn, high-M nozzle source, (conditions?) 


\section{Miller \& Kusch TOF Results}

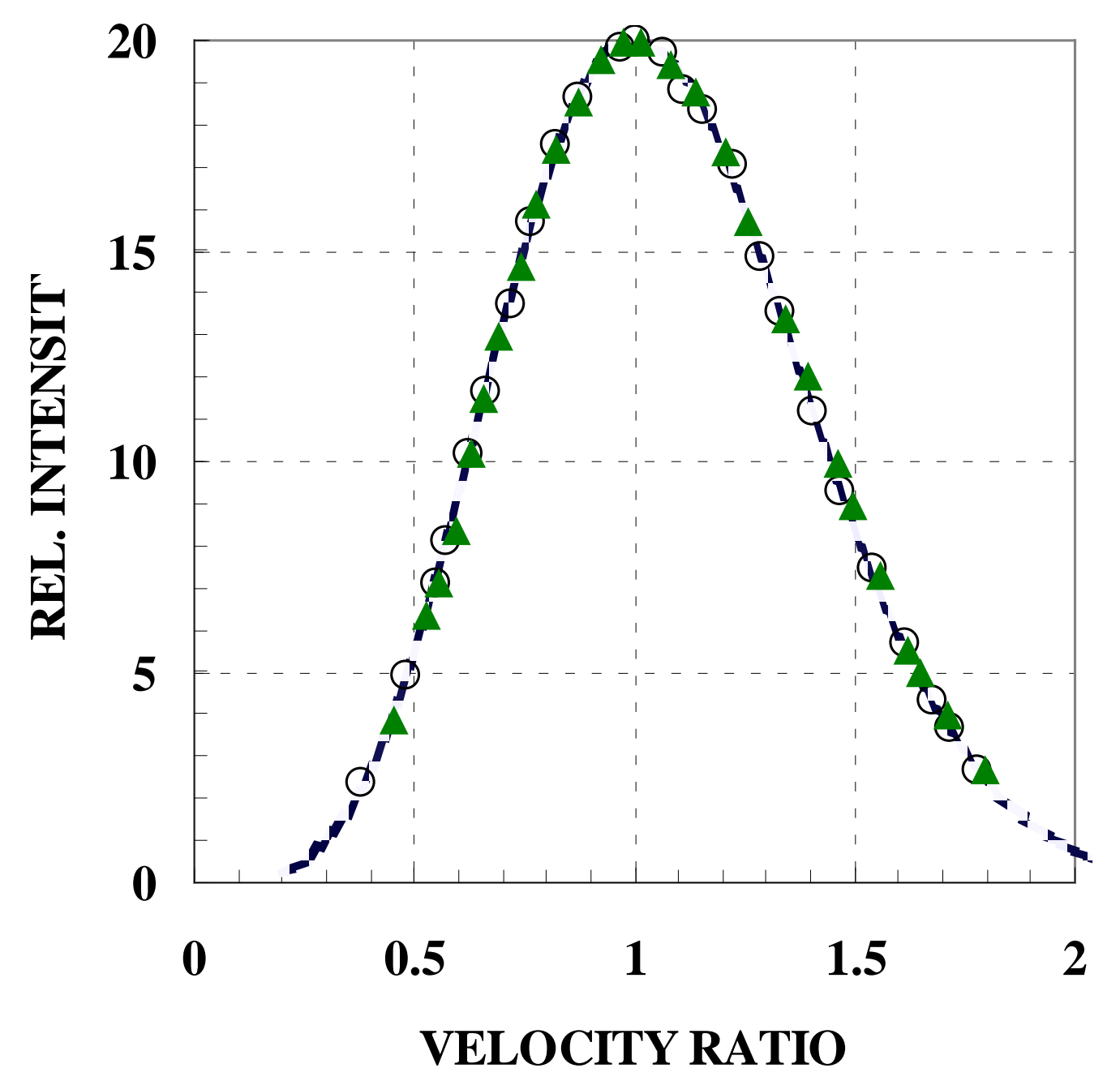




\section{MB TOF Results}

- For effusion, Miller \& Kusch determined

$$
\rho \propto \alpha^{4} e^{-\alpha^{2}}
$$

- For high-Mach number, Anderson \& Fenn found

$$
\rho \propto \alpha^{4} e^{-z^{2}}
$$

- Both developments are reproduced by pulse source model 


\section{Pulsed Laser Ablation Simulation}

- Bykov et al. modeled pulsed laser ablation (PLA) process for heating and vaporization of niobium due to varying laser fluence levels $\left(4-6 \mathrm{~J} / \mathrm{cm}^{2}\right)$

- Used DSMC to simulate subsequent vapor release

- Noted that effluent angular distributions changed from approximately spherical expansions to ones more focused along surface normal for early periods

- Relaxed to spherical expansions as time and rarefaction levels increased

- Presented centerline density using

$$
f_{n} \equiv \frac{n t^{3}}{n_{A V} \tau_{L}}
$$




\section{PLA - Model Development}

- Pulse mode model suggests that

$$
n t^{3} \propto f_{n} \propto \alpha e^{-z^{2}}
$$

- Maximized for

$$
\alpha=\frac{s+\sqrt{s^{2}+2}}{2}
$$

- For effusive flow, occurs for $\alpha=1 / \sqrt{2}$

- For non-zero $s$, critical $\alpha$ increases 


\section{PLA Model Comparison}

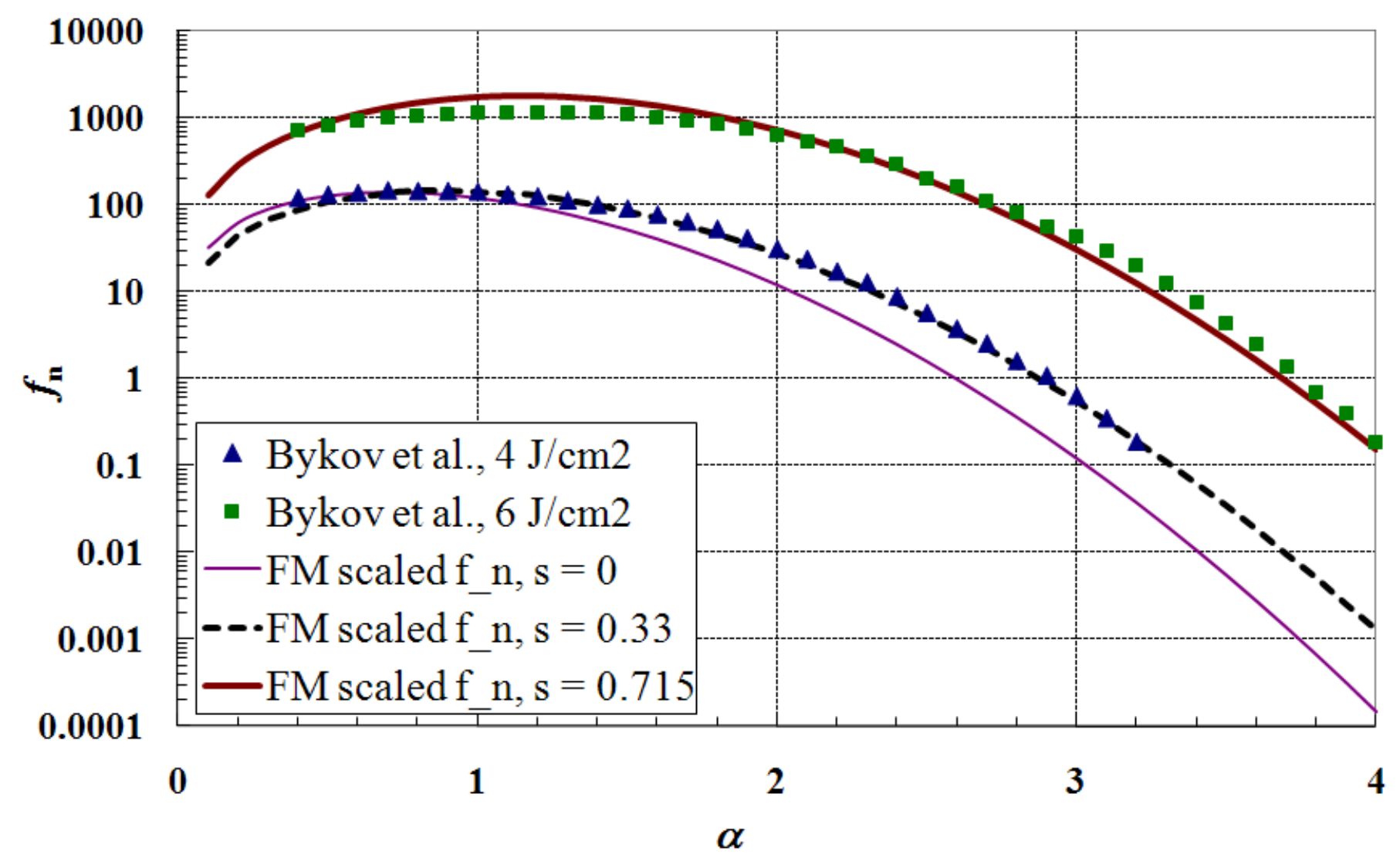




\section{Outline}

- Introduction

- Model Development

- Selected Model Comparisons

- Selected Satellite Applications

- Formation Flying Interaction

- LADEE Lunar Surface Impingement

- Concluding Remarks 


\section{“Afternoon Constellation”}

- International effort to operate heterogeneous satellite group to make near-simultaneous measurements over defined ground track

- Was to feature seven satellites, including OCO and Glory

- Sun-synchronous orbit

- $705 \mathrm{~km}$ altitude

- One minute separation $\approx 450 \mathrm{~km}$

- Each S/C features aft-facing $\mathrm{N}_{2} \mathrm{H}_{4}$ Attitude Control System (ACS) thrusters

- Atmosphere (MSISE-90 model)

- 99+\% atomic oxygen

$-n_{\infty} \approx 2.1 \times 10^{11} / \mathrm{m}^{3}( \pm 5 \times)$ 


\section{A-Train Configuration}

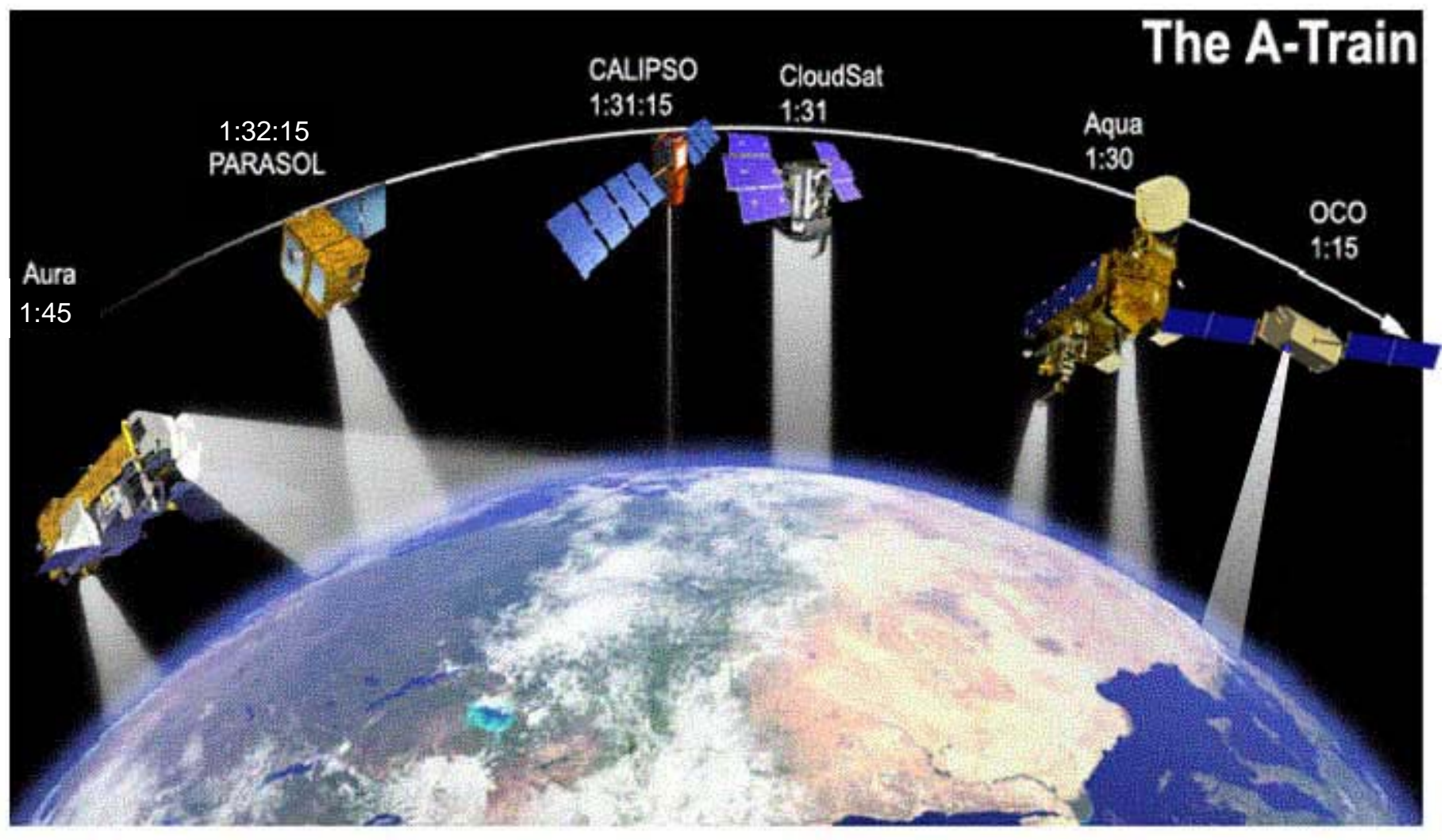




\section{Satellite Formation Flying (FF) Interaction Example}

- Program managers sometimes voice concern that thruster operations from one spacecraft may create non-negligible effects on others

- Schedule and cost always at a premium

- Combination of thruster pulse durations and separation distances may mean impingement events should be modeled in a transient mode

- In particular, estimate CloudSat ACS operation effects on CALIPSO

- 1- $\mathrm{N} \mathrm{N}_{2} \mathrm{H}_{4}$ units operating for $19 \mathrm{~s}$ (step functions, square wave)

- CALIPSO trails by $15 \mathrm{~s}(\approx 110 \mathrm{~km})$ 


\section{FF Thruster Parameters}

- A-Train S/C ACS feature sets of aft-facing monopropellant hydrazine thrusters

- Mass flow rate $=2.0 \mathrm{~g} / \mathrm{s}$ per $\mathrm{lb}_{\mathrm{f}}$ thrust, $V_{\mathrm{e}}=2.2 \mathrm{~km} / \mathrm{s}$

- Plume species based on $65 \% \mathrm{NH}_{3}$ decomposition

$-\mathrm{H}_{2} \mathrm{O}$ included as fuel impurity at $0.5 \%$ mass fraction

- Neglected

- Gravitational effects

- Scattering by ambient atmosphere

- CALIPSO should encounter peak impingement fluxes roughly 50 - 70 s after CloudSat commences a 19 s operation 


\section{CloudSat/CALIPSO_Various Fluxes}
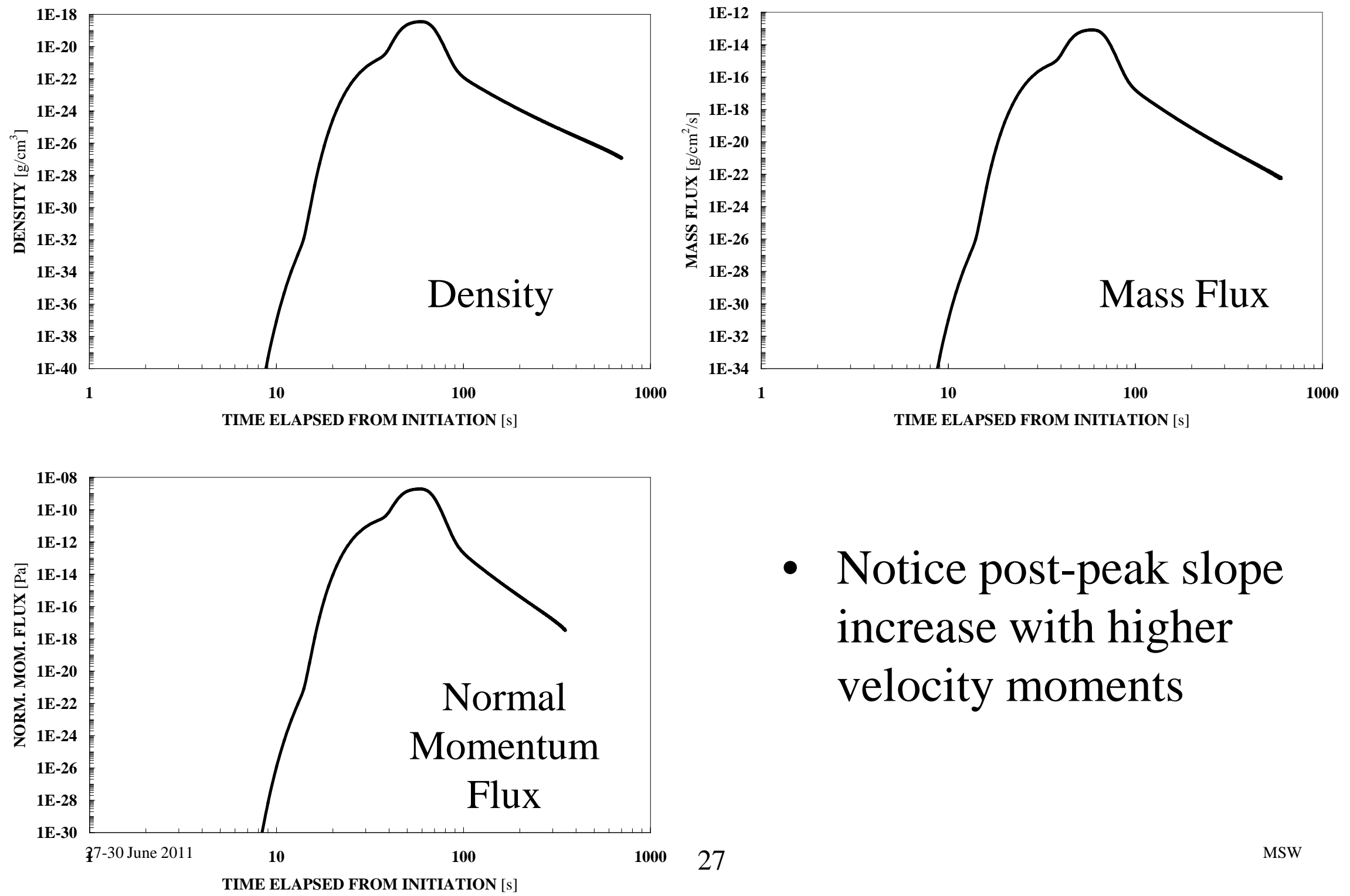

- Notice post-peak slope increase with higher velocity moments 


\section{Example-CloudSat/CALIPSO, GMW}

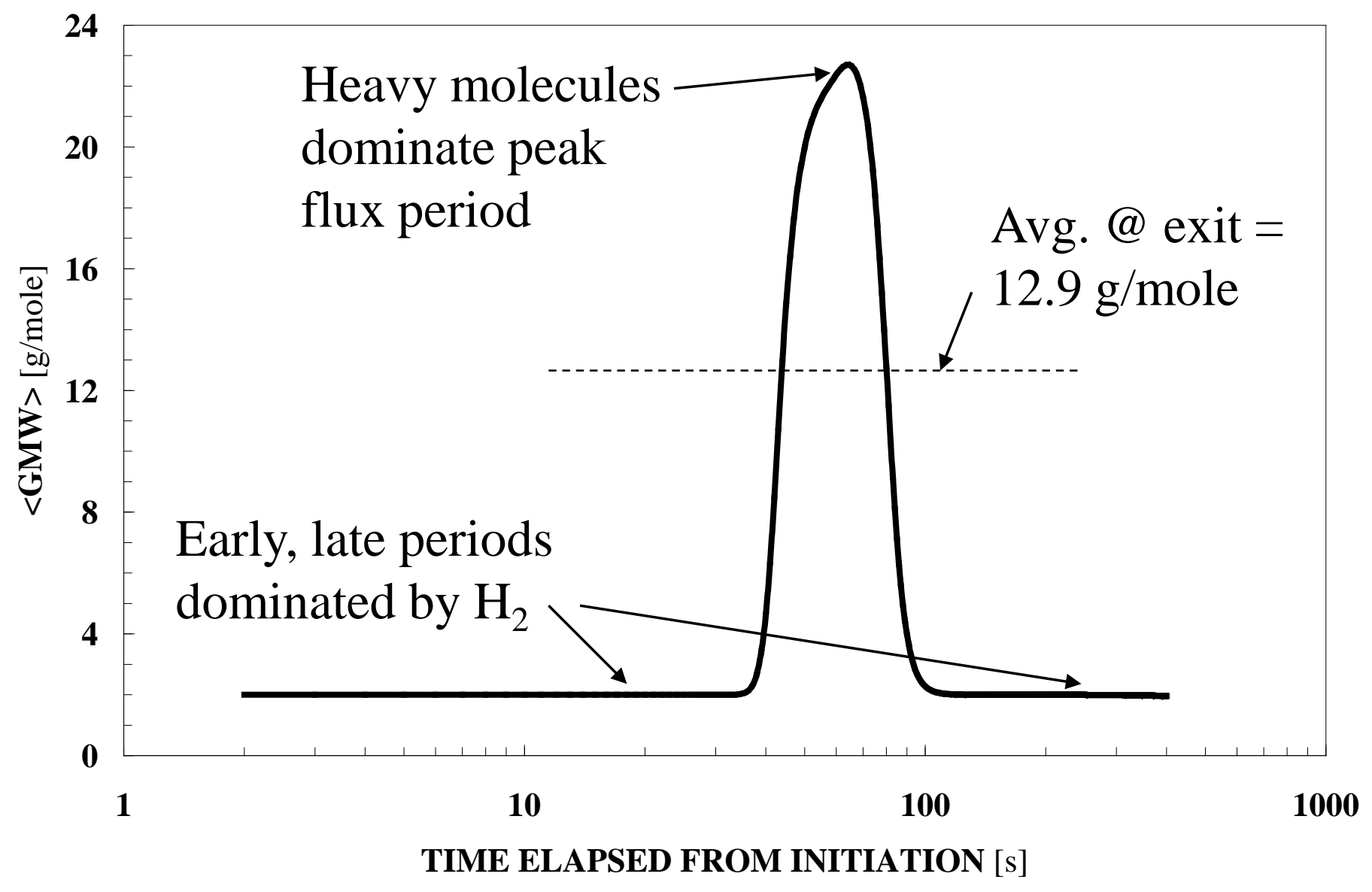




\section{Induced Environment Effects}

- Depending on circumstances, thruster operation induced environments might not be insignificant

- CloudSat $\rightarrow$ CALIPSO ( $\Delta t=15 \mathrm{~s}$ ), single thruster:

$$
\rho_{\text {max }}=3.5 \times 10^{-19} \mathrm{~g} / \mathrm{cm}^{3}
$$

- Two thrusters $\rightarrow$ peak density $\sim 12$ percent of ambient levels 


\section{LADEE Introdction (1 of 2)}

- Lunar Atmosphere Dust Environment Explorer (LADEE)

- Collect data regarding lunar atmosphere (gases, dust) before alteration due to future exploration activities

- Features include

- Operational period $~ 100$ days

- Variety of orbits (elliptical, circular)

- Nominal $=50 \mathrm{~km}$, circular

- As low as $20 \mathrm{~km}$, circular

- Variety of orientations used for making measurements, communicating with Earth

- Lunar atmosphere is so rarefied it's referred to as an "exosphere"

- Essentially free-molecule conditions 


\section{LADEE Introduction (2 of 2)}

- Instruments include Neutral Mass Spectrometer (NMS)

- Design is sensitive enough to detect $~ 100$ molecules/ $/ \mathrm{cm}^{3}$

- NMS measurement sensitivity drives many LADEE contamination control requirements

- Causes consideration of unusual scenarios

- Outgassing

- Attitude Control System (ACS) thruster plume influence 


\section{LADEE Spacecraft}

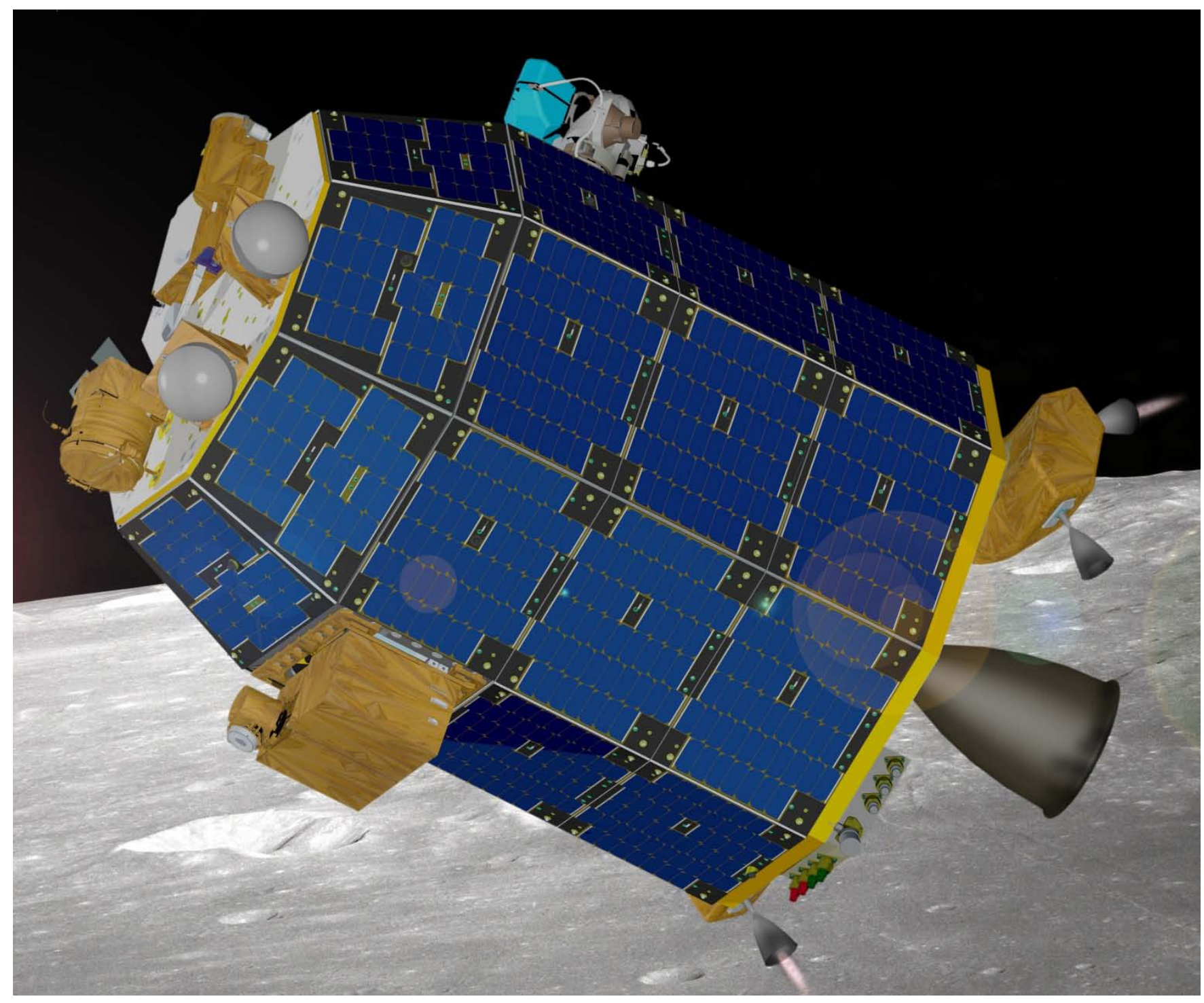




\section{Schematic Diagram for Study}

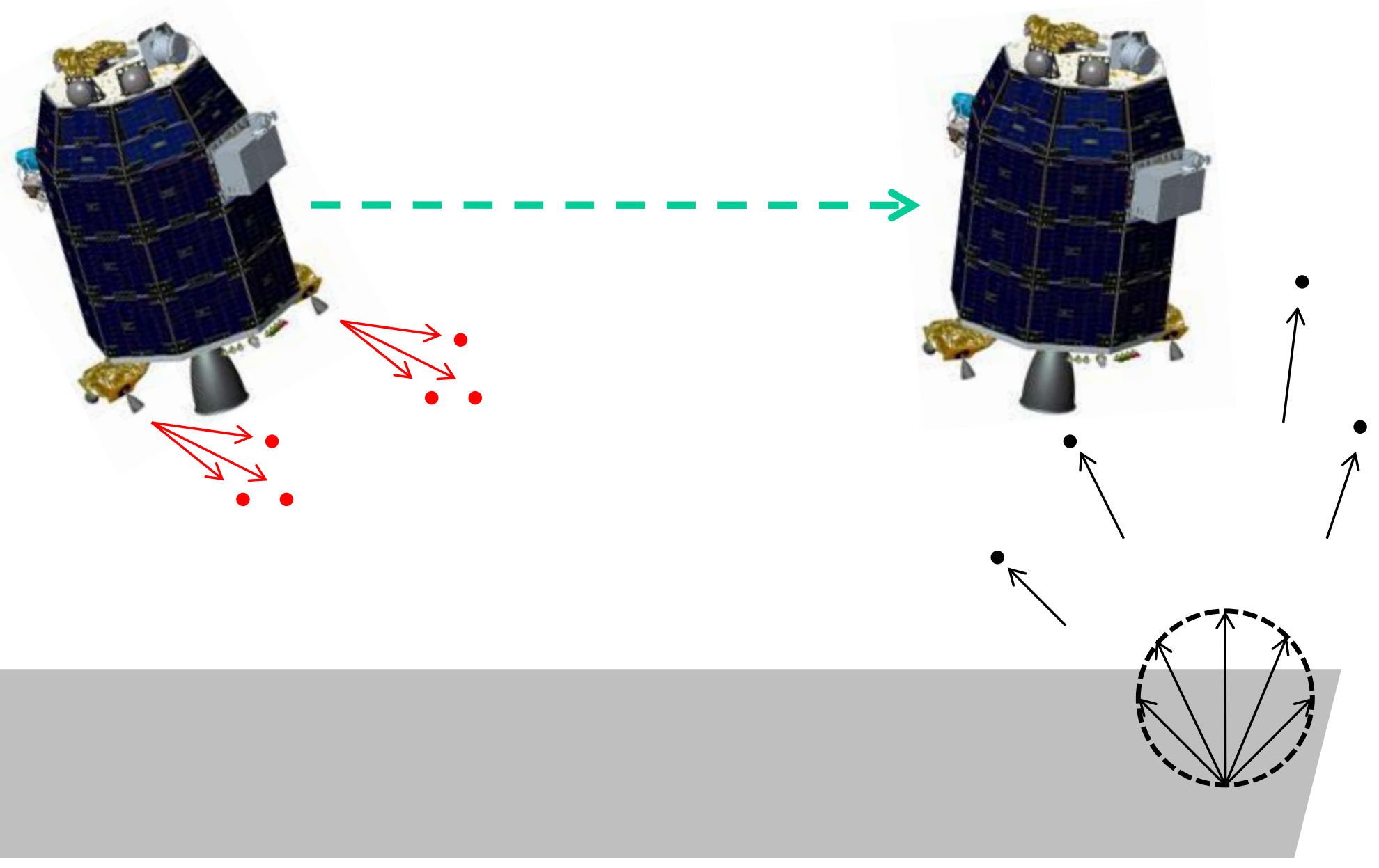




\section{LADEE Case Study Objective}

- Appeared conceivable NMS could measure ACS plume gases reflected from sunlit, impermeable lunar surface

- At minimum altitude

- Measurement would be maximized

- Gravitational influence minimized ("short" time-of-flight situation)

- Could use to verify aspects of thruster plume modeling

- Model the transient disturbance to NMS measurements due to ACS gases reflected from lunar surface

- Observe evolution of various model characteristics as measured by NMS

- Species magnitudes, TOF measurements, angular distribution, species separation effects 


\section{LADEE Test Case Conditions}

- Minimum altitude (20 km, circular)

- NMS faces ram direction

- Forward-facing ACS thruster pair

- Operates for $1 \mathrm{~s}, 20^{\circ}$ below horizontal

- Ignore changes in spacecraft altitude (use pulse mode)

- Units: 5-lb $\mathrm{f} \mathrm{MMH} / \mathrm{MON}-3$

$-V_{\mathrm{e}} \approx 3.0 \mathrm{~km} / \mathrm{s}, T_{\mathrm{e}} \approx 550 \mathrm{~K}$

\begin{tabular}{|c|c|c|c|c|c|}
\hline Species & $\mathbf{N}_{\mathbf{2}}$ & $\mathbf{H}_{\mathbf{2}} \mathbf{O}$ & $\mathbf{C O}$ & $\mathbf{C O}_{\mathbf{2}}$ & $\mathbf{H}_{\mathbf{2}}$ \\
\hline$Y_{i}$ & 0.43 & 0.29 & 0.18 & 0.086 & 0.016
\end{tabular}

- Particularly interested in water vapor influence 


\section{LADEE_Lunar Impingement: Surface Density}
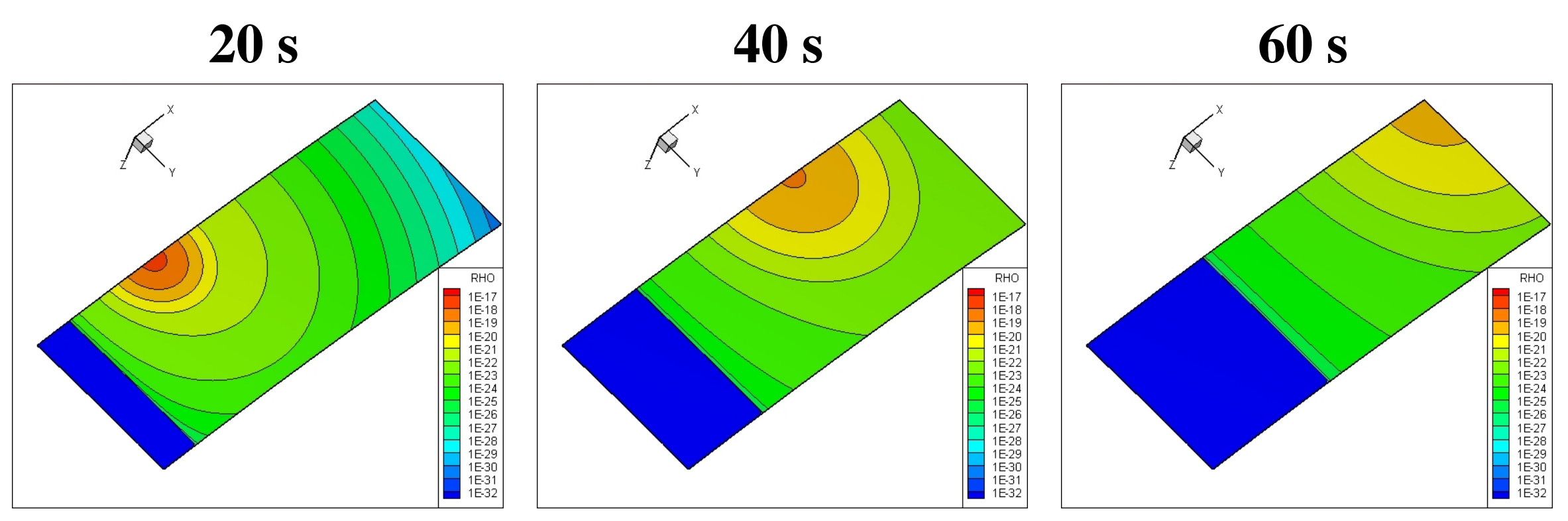


\section{LADEE-Lunar Impingement: NMS Species Density Estimates}

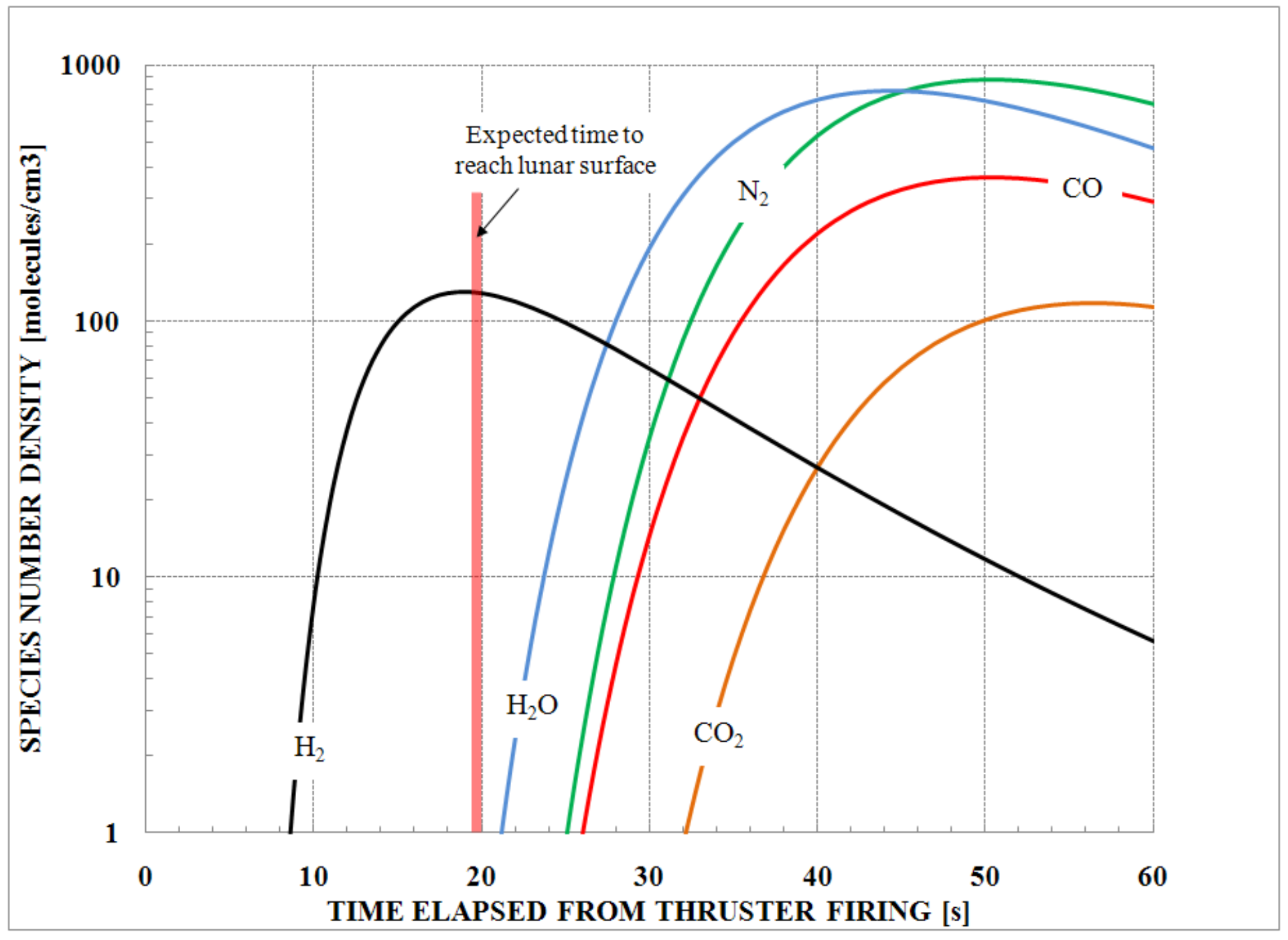




\section{Nozzle Exit Temperature $T_{\mathrm{e}}$ Influence}

- Elapsed time for peak species mass fluxes to reach lunar surface occurred quicker than expected based on $V_{\mathrm{e}} \sin 20^{\circ}$

- Time derivative of mass flux equations $\left(\Phi \propto t^{-D}\right)$ indicates

$$
t_{\max f l u x}=\frac{2 \beta r}{w\left(1+\sqrt{1+\frac{2 D}{w^{2}}}\right)}
$$

- For $w=s$ on the plume centerline, $t_{\max \text { flux }} \rightarrow r / V_{\mathrm{e}}$ as $s \rightarrow \infty$

- For finite $s$, this period is always shorter

- Consequence of thermal energy component 


\section{Concluding Remarks}

- A FM model has been created for estimating thruster plume effects

- Ongoing verification effort for a wide variety of conditions demonstrates utility of this approach

- Results characterized by

- Transient effects

- Superposition

- Species separation

- Long-term persistence of lightweight species 


\section{Extra Materials}




\section{Example-Approach to Steady State (cont.)}

- Upstream locations tend to experience longer transients than downstream

- Review variable $z$

- Highest angles produced upstream, lowest $\cos \theta$ values, longest transient influences

$z \equiv \alpha-w=\frac{\beta r}{t}-s \cos \theta$

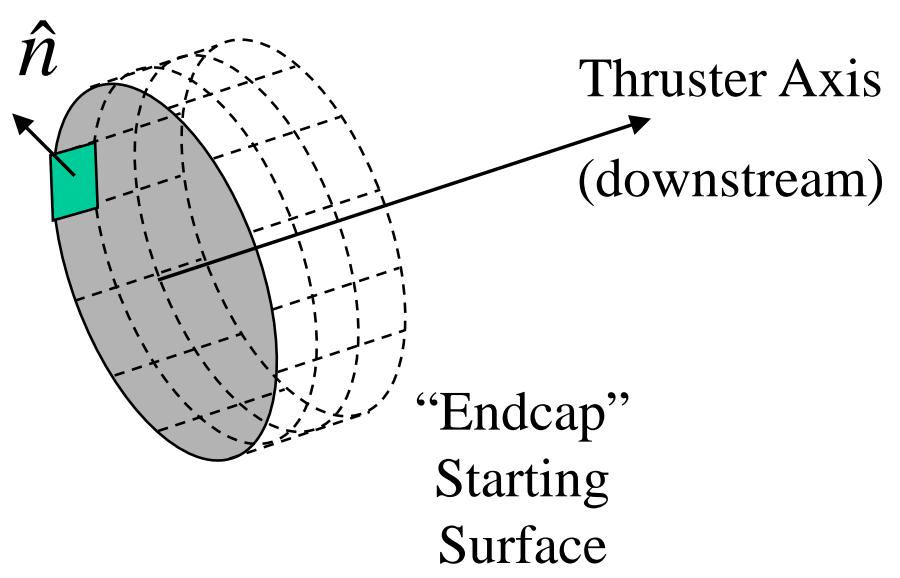




\section{Example-Impingement Fluxes}

ENHANCED FLYING FORMATION THRUSTER INTERACTIONS TOTAL MASS FLUX EXPERIENCED BY TARGET SPACECRAFT

DUE TO EOS-AQUA (single $1-\mathrm{lb}_{\mathrm{f}}$ thruster, $100 \mathrm{~s}$ )

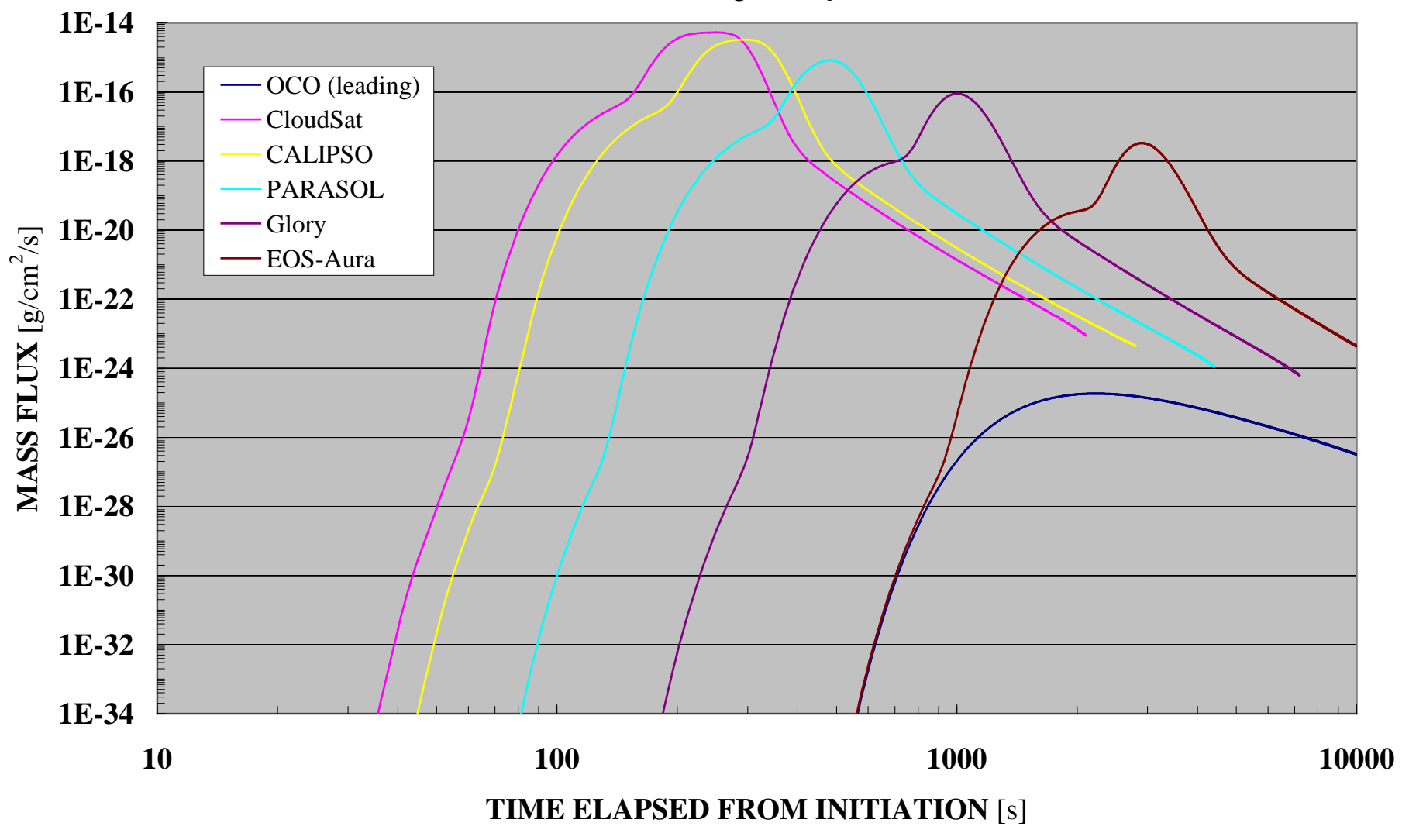




\section{Example-Impingement Fluxes, <GMW >}

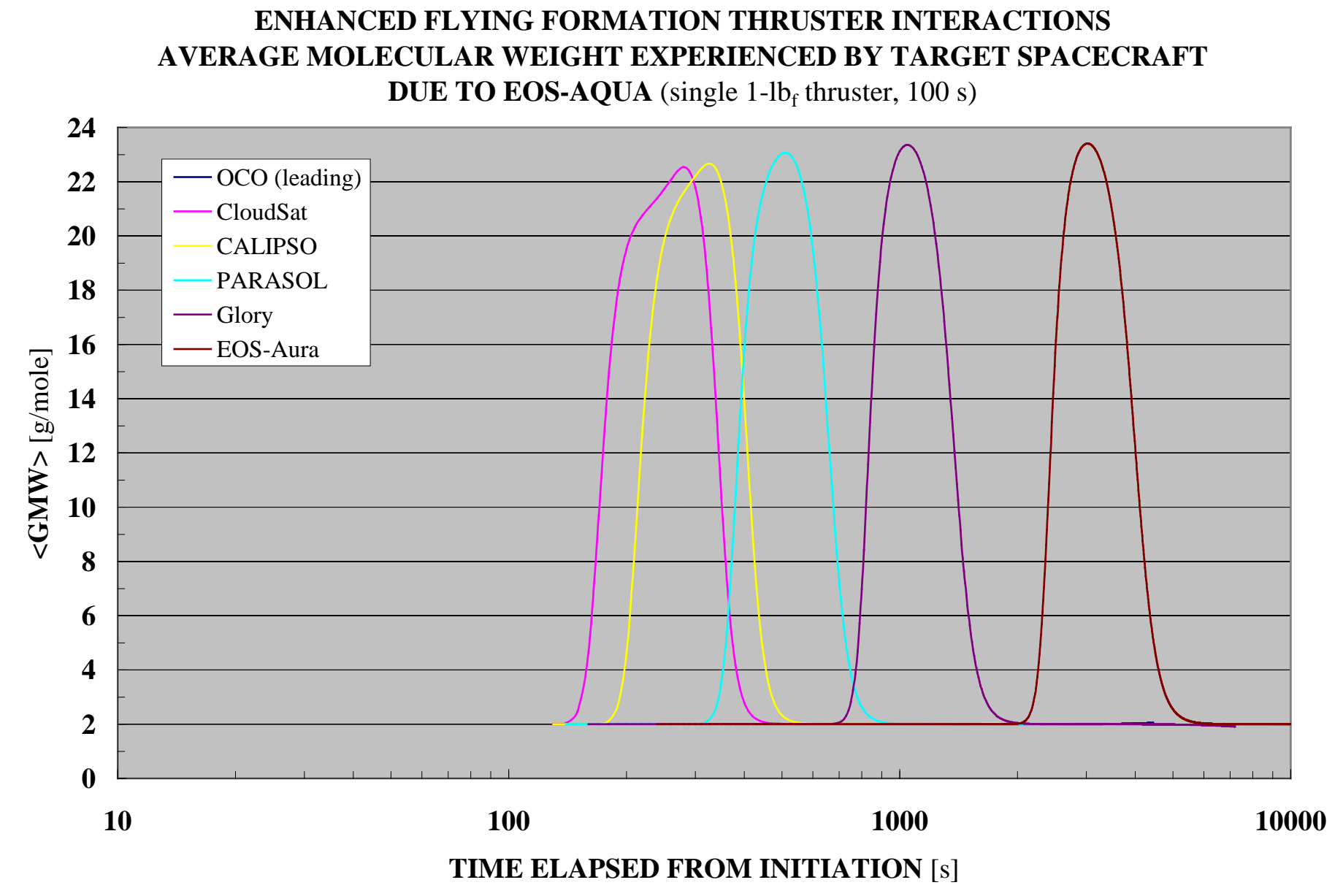




\section{Example-Impingement Fluxes (cont.)}

- Compare OCO \& Aura environments

- Early behavior dominated by wave-like term $\alpha$

- Essentially all $\mathrm{H}_{2}$

- Diverges due to effect of bulk motion downstream

- Absent upstream

- Still essentially $\mathrm{H}_{2}$

- Extra downstream hump due to increasing presence of other plume species

- Maximum fluxes occur near time expected due to bulk velocity

- Only $\mathrm{H}_{2}$ left lingering at longest times 


\section{Example-Pulse, Step Comparison}

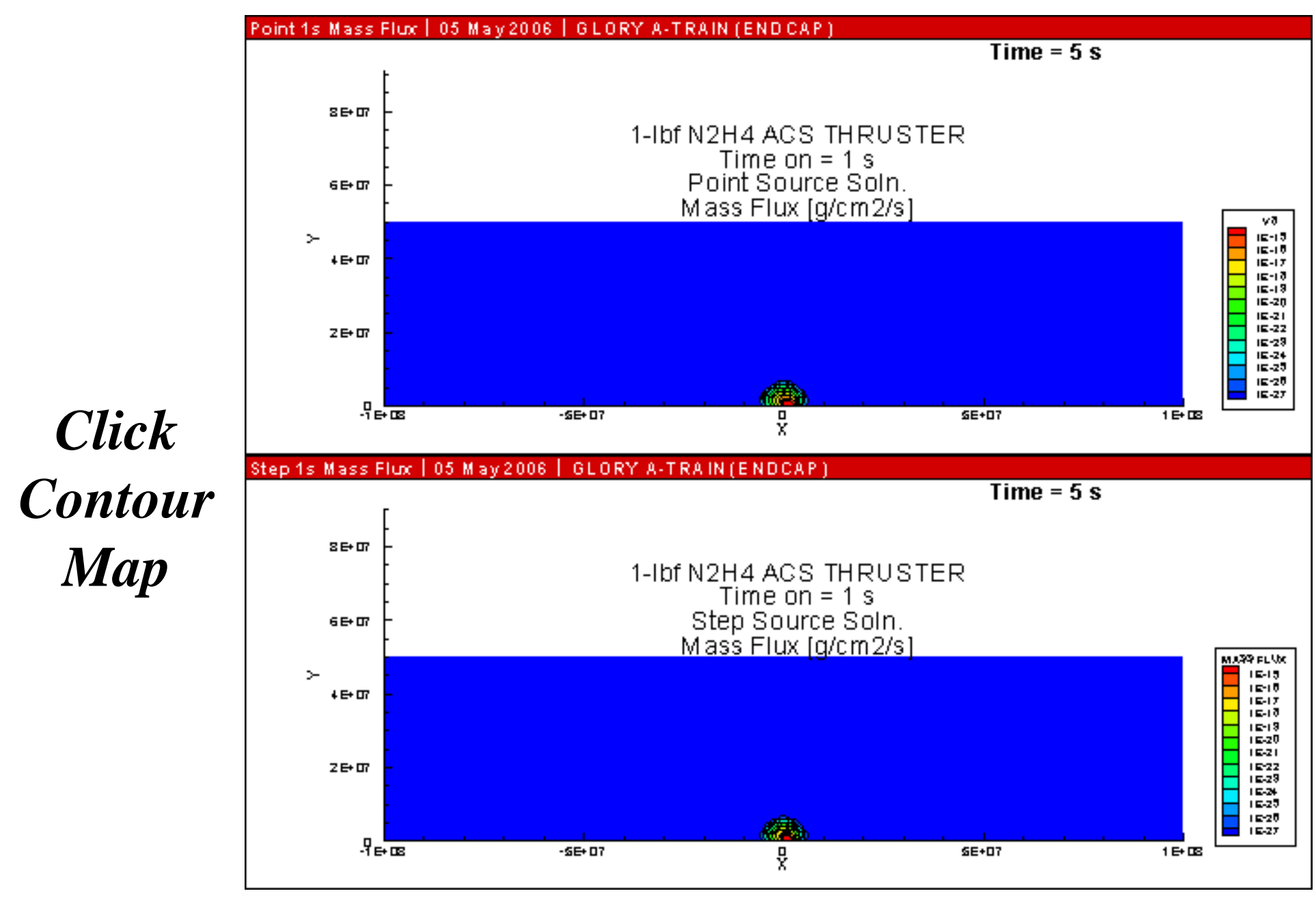




\section{Example-Approach to Steady-State}

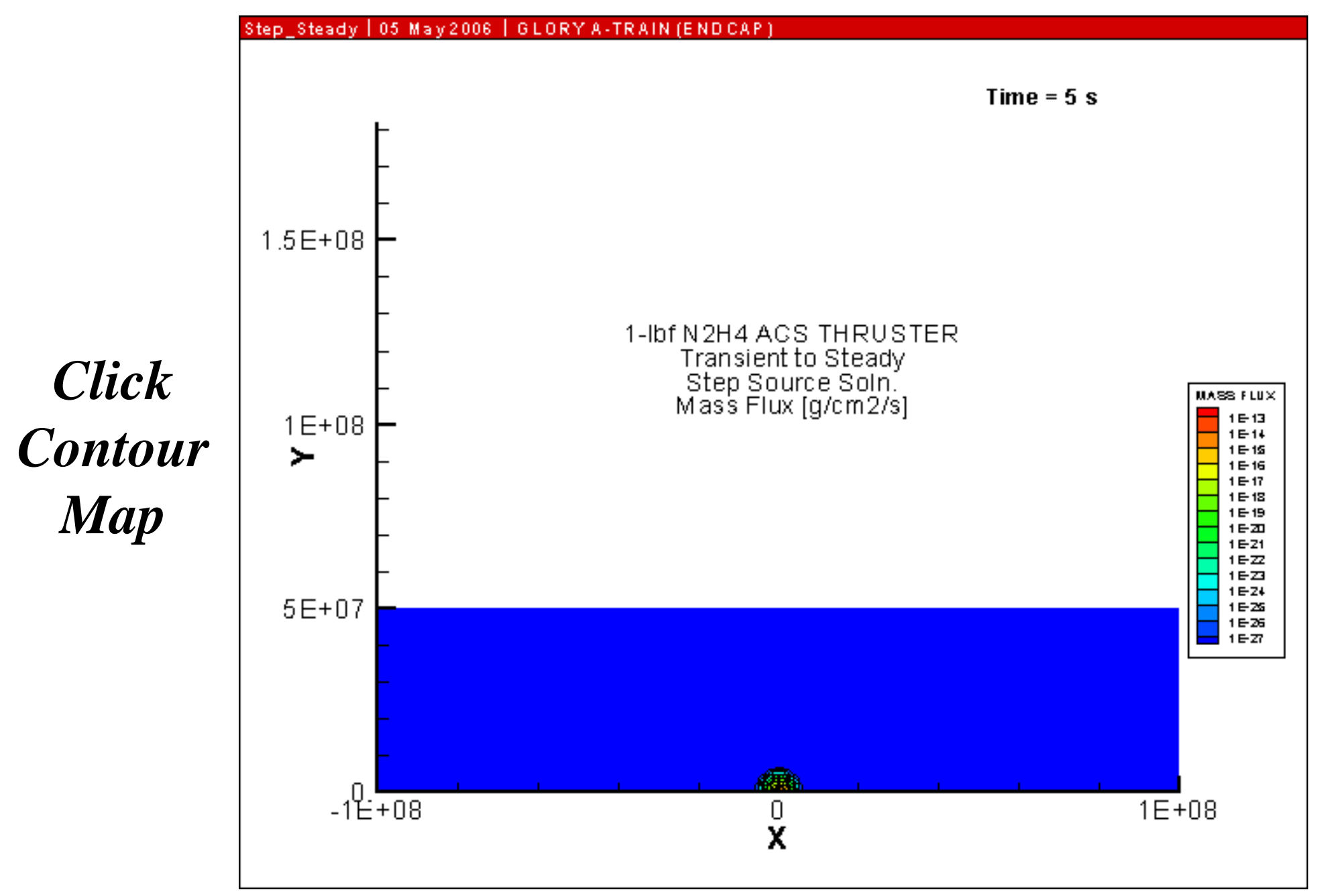




\section{Example-100 s Operation}

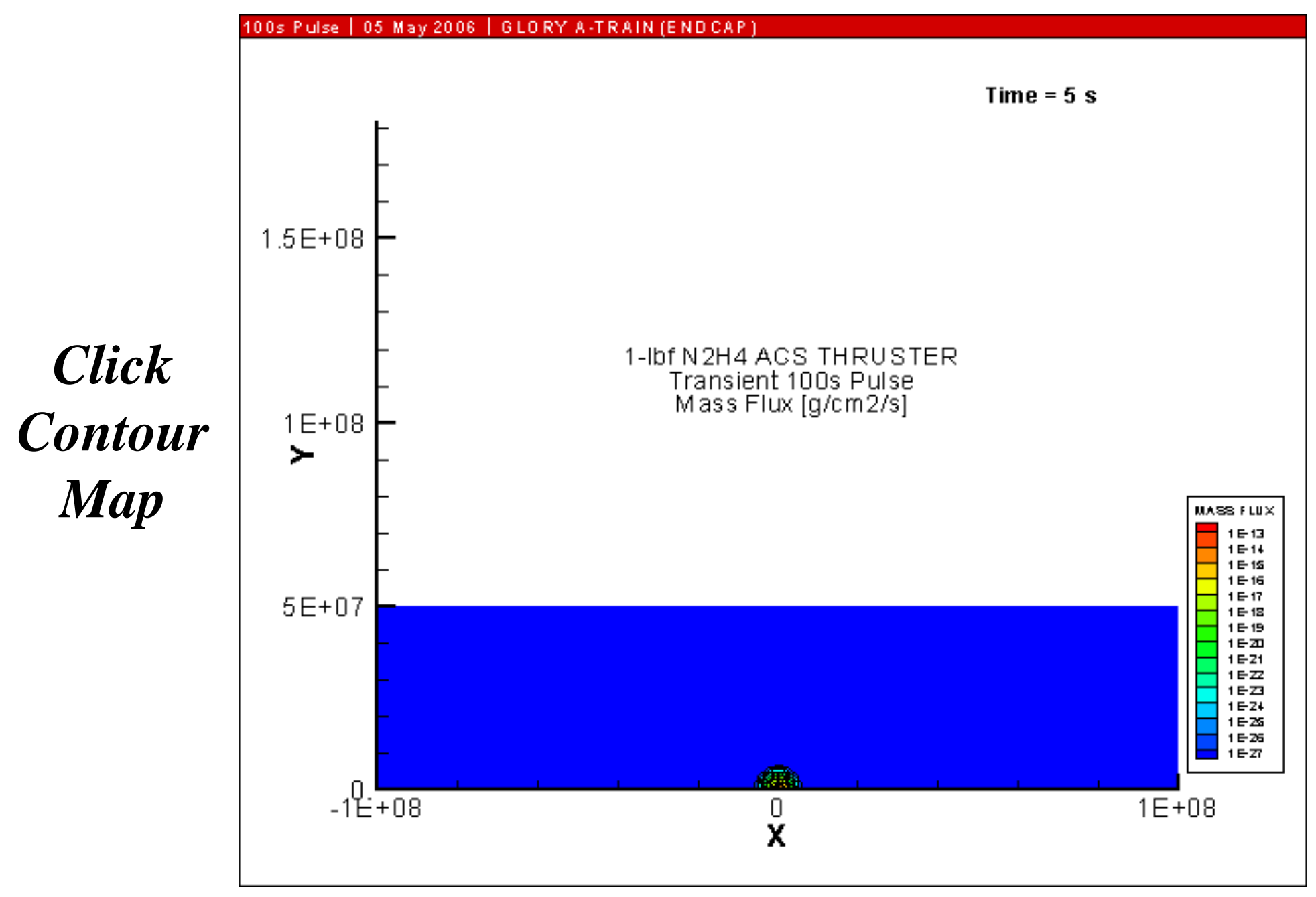




\section{CloudSat/CALIPSO—Energy Fluxes}
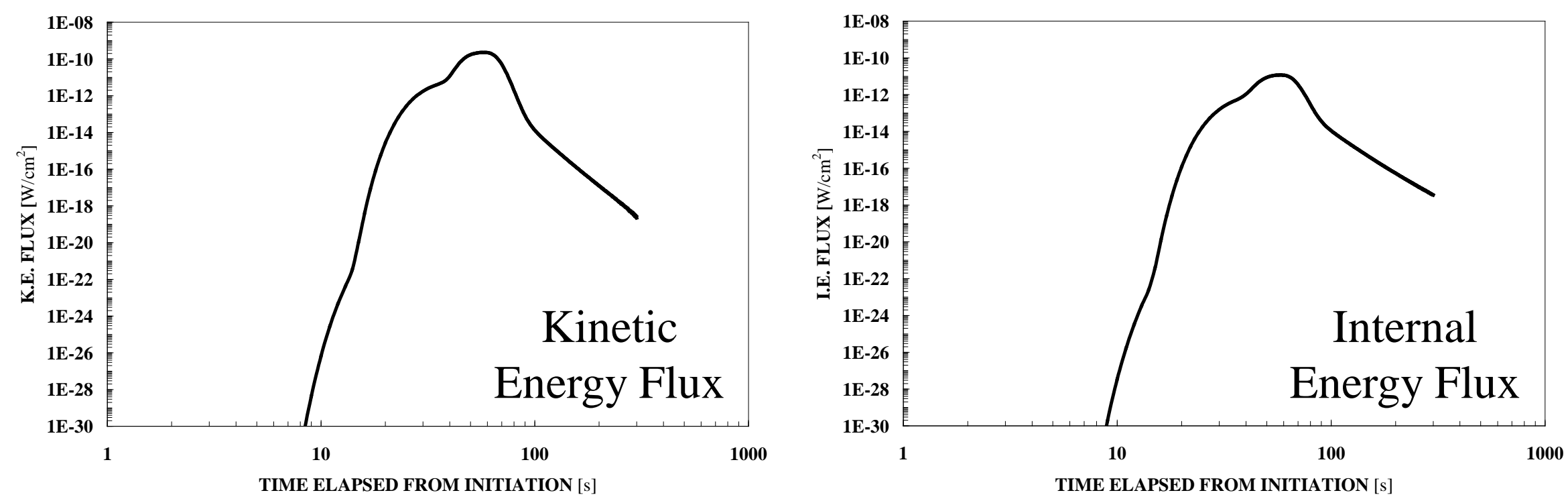

- Long-term, model predicts eventually higher IE flux than KE flux

- IE flux created as product of IE/mass and mass flux

- Not actually realized (radiative loss of IE with time) 


\section{CloudSat/CALIPSO—Velocity}

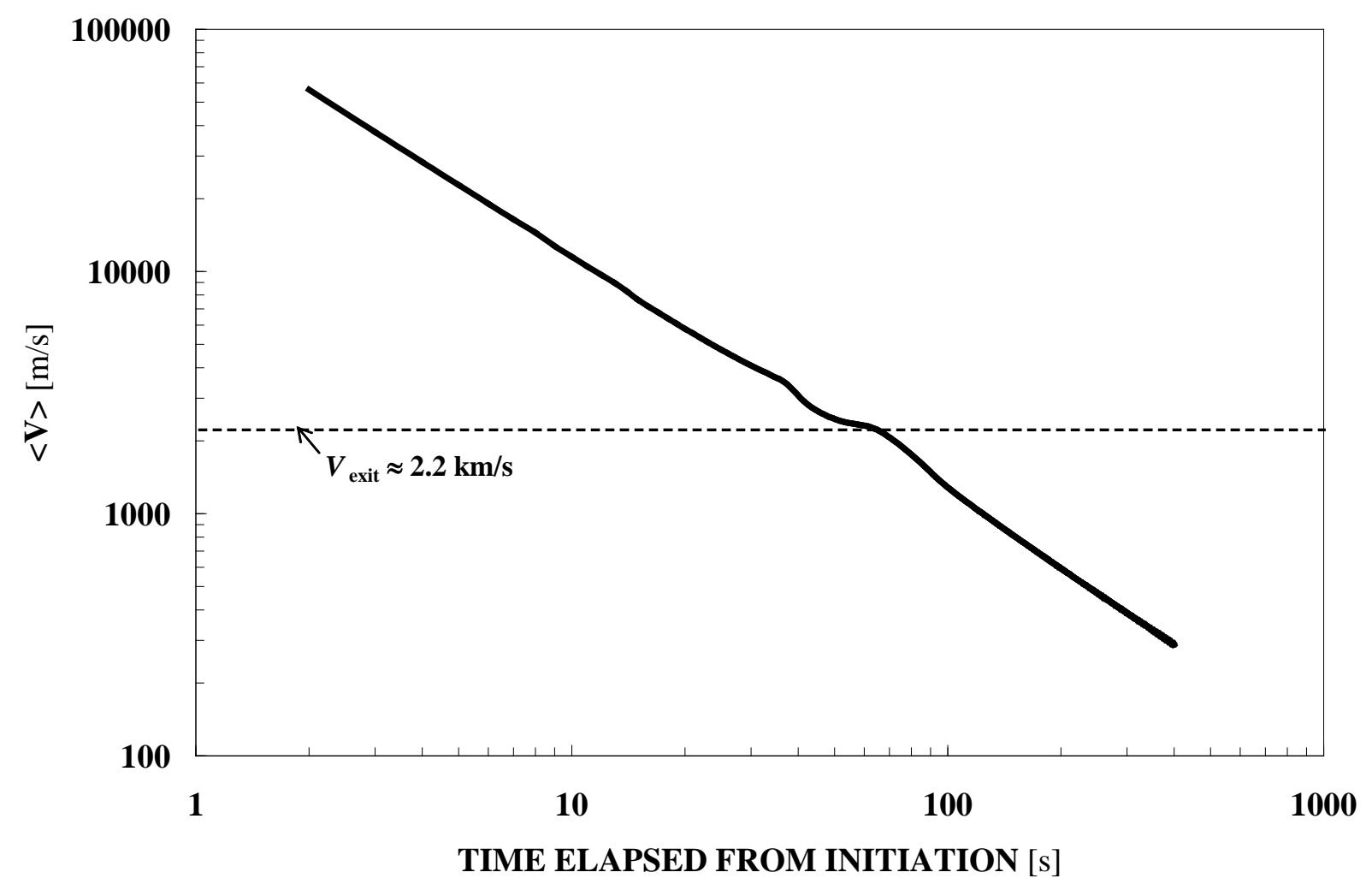

- Not exactly given by $r=v t$; deviations evident around $V_{\text {exit }}$

- Effect of finite pulse length still significant

- Superposition effect due to multiple species 